Nuclear Physics B161 (1979) 493-532

(c) North-Holland Publishing Company

\title{
THE FERMION MASS SCALE AND POSSIBLE EFFECTS OF HIGGS BOSONS ON EXPERIMENTAL OBSERVABLES
}

\author{
H.E. HABER *, G.L. KANE and T. STERLING \\ Physics Department, University of Michigan, Ann Arbor, Michigan 48109, USA
}

Received 22 December 1978

(Revised 5 July 1979)

\begin{abstract}
We consider a conventional SU(2) $\otimes \mathrm{U}(1)$ gauge theory with two (or more) Higgs doublets, but with the fermion mass scale determined by the vacuum expectation values of the Higgs particles rather than determined only by widely differing Higgs couplings. Such an alternative to the standard theory cannot be excluded by current data; the Higgs-fermion coupling is allowed to be at least 70 times that of the standard WeinbergSalam theory. In such a model, one has the possibility of observing large and interesting effects due to the Higgs particles in the theory. These include decays of heavy quarkonium states and Drell-Yan production of $\ell^{+} \ell^{-}(\ell=e, \mu, \tau)$. Restrictions due to charged Higgs scalar currents (in $\pi, \mu$ and $\beta$ decay and in neutrino production) take unexpected forms and are not too stringent. The best place to search for a Higgs is in $\mathrm{K}^{+} \mathrm{N} \rightarrow \mu^{+} \mu$ X (subsect. 3.5).
\end{abstract}

\section{Introduction}

For two reasons, we want to examine alternatives to the usual Higgs sector in otherwise conventional gauge theories of weak and electromagnetic interactions. First, if nature is described by a spontaneously broken gauge theory, it is the physical Higgs particles and the phenomena associated with them that are the qualitatively different features which need to be confirmed by experiments [1-3]. The Higgs bosons are fundamental scalar particles in the lagrangian of the theory, and give scalar currents. Unfortunately, in the standard theory with one Higgs doublet and one (neutral) physical Higgs boson both the particle and any phenomena associated with its interactions are likely to be almost unobservable. The problem is that its coupling to fermions is proportional to their mass $\left(m_{\mathrm{f}}\right)$. Consequently, the Higgs contribution to low-energy observables is typically suppressed by powers of $m_{\mathrm{f}} / m_{\mathrm{W}}$. Similiarly, its production cross sections [2] are very small, suppressed by similar factors in most processes. If produced, it decays into the heaviest available fermions

\footnotetext{
${ }^{\star}$ Current address: Lawrence Berkeley Laboratory, Berkeley, Ca 94720.
} 
(e.g., $\tau^{+} \tau^{-}, c \bar{c}$ or $b \bar{b}$ ) which themselves have multibody decays, so the $\mu^{+} \mu^{-}$mode which could give a mass peak has a tiny branching ratio. If the Higgs is sufficiently light, detecting [4] its presence via the decay of a heavy quarkonium vector boson, $\mathrm{V} \rightarrow \gamma \mathrm{H}$, is the only place where some optimism seems warranted. If the Higgs is very heavy, it could only be seen through its effects on interactions. It could indeed lead to large weak interaction effects at high energies $[5,6]$, but these would be screened off from experimental observation $[5,7]$ by the small coupling to fermions.

A minor generalization of the Higgs sector to two doublets, which we will consider, can change the entire situation. Then it is possible to have much larger effects of Higgs bosons on low-energy interactions and on high-energy weak interactions, larger production cross sections, and significant widths for decay into $\mu^{+} \mu^{-}$. If nature were similar to such a generalization it would be relatively easy to observe Higgs bosons, their effects, or both.

A second reason to consider an alternative Higgs sector stems from the recent successes of the standard $S U(2) \otimes U(1)$ theory. From charged and neutral current phenomenology there is extensive confirmation of the predictions of the standard theory and most alternative suggestions have been excluded $[8,9]$. The situation concerning parity violation in the electron-nucleon interaction is still confused experimentally but the observed asymmetry [10] in polarized electron-nucleon scattering is in agreement with the standard theory. Hopefully, the experiments in atomic hydrogen and deuterium [11] will soon clarify the situation. Although extensive factorization tests [12] have yet to be performed, so far data is consistent with one $Z^{0}$ and no right-handed charged currents, and in detail with the standard theory. The evidence suggests that only Higgs doublets occur, since the ratio of neutral to charged current coupling $\left(m_{\mathrm{W}}^{2} / m_{Z}^{2} \cos ^{2} \theta_{\mathrm{W}}\right)$ is unity for a theory with only Higgs doublets [13] and is observed [8] to be $0.98 \pm 0.05$. Thus, apart from the possibility of several Higgs doublets, the existing data constrain any theory to be essentially equivalent to the simple standard theory in the aspects accessbile to present day experiments.

There have been many alternative Higgs sectors proposed. Usually these have a sector equivalent to the standard theory and differ in largely unobservable ways. We want to consider a variation which is qualitatively different from most others with a large number of observable effects and consequently easy to exclude experimentally. If it can be excluded it will strengthen the interpretation that the standard theory with one Higgs doublet is basically correct.

The approach we take can be summarized as follows. If there are two Higgs doublets then two different vacuum expectation values are available to give masses to the vector bosons and fermions. Our basic assumption is that the very different mass scales of the fermions and of the vector bosons are set by different Higgs vacuum expectation values. Then the theory has a (dimensionless) parameter

$$
\beta=v / V,
$$


where $v$ is the small vacuum expectation value which gives mass to the fermions, and $V$ is the large vacuum expectation value which sets the mass scale for the vector bosons.

The coupling to fermions is then

$$
g_{\mathrm{f}}=g m_{\mathrm{f}} / 2 \beta m_{\mathrm{W}} .
$$

Note that if $\beta$ can be of order $m_{\mathrm{f}} / m_{\mathrm{W}}$, we can have $g_{\mathrm{f}} \simeq g$ and many large effects can occur. We have looked in detail to see how experiment restricts the Higgs-fermion couplings. While there are restrictions (e.g., $\beta$ is unlikely to be less than 0.015 ) there is still room for Higgs effects which are substantially larger than in the standard model without being inconsistent with experiment at present. Numerous tests will be available.

There are five physical Higgs particles in this model. One, denoted by $\phi$, has properties similar to the conventional neutral Higgs boson of the one-doublet case and leads to no new phenomena. Two others are neutral, and two charged, as summarized below ( $g_{\mathrm{f}}$ as is defined in eq. (2)):

$\begin{array}{llll}\text { Particle } & J^{P} & \text { Mass } & \text { Interaction } \\ \phi & 0^{+} & \text {heavy } & \sim \beta g_{\mathrm{f}} \\ \mathrm{h}^{0} & 0^{+} & \text {light } & \sim g_{\mathrm{f}} \\ \mathrm{H}^{0} & 0^{-} & \text {heavy } & \sim g_{\mathrm{f}} \\ \mathrm{H}^{ \pm} & 0^{+}, 0^{-} & \text {heavy } & \sim g_{\mathrm{f}}\end{array}$

The $W^{ \pm}$mass in the model is of order $g V$ and the heavy Higgs $(H)$ masses are of or$\operatorname{der} \sqrt{\lambda} V$, where $\lambda$ is a typical coefficient in the Higgs potential; $\lambda$ is unknown but could be of order $g^{2}$. The light Higgs $\left(h^{0}\right)$ has mass of order $\beta \sqrt{\lambda} V$ so its mass could be small compared to the heavy Higgs mass if $\beta<<1$. The interactions of $h^{0}$ could contribute to low energy observables (see below). The presence of $\mathrm{H}^{0}$ (and $\mathrm{H}^{ \pm}$) could lead to large, observable weak interaction effects at very high energies since it has large coupling to fermions if $\beta<1$ [7]. Since the Higgs masses depend on the coefficients in the Higgs potential they are not determined, but the light-heavy distinction will be maintained unless the coefficients vary widely.

The Higgs-fermion lagrangian for leptons, in the model, is $\left(\psi_{\mathrm{L}}=\frac{1}{2}\left(1+\gamma_{5}\right) \psi\right)$

$$
\begin{aligned}
-\mathcal{L}= & \frac{m_{\ell} g}{2 \beta m_{\mathrm{W}}}\left[\overline{\ell \ell} \phi \sin \alpha+\bar{\ell} \ell h^{0} \cos \alpha-i \bar{\ell} \gamma_{5} \ell H^{0} \cos \beta\right] \\
& -\frac{m_{\ell} g}{2 \sqrt{2} \beta m_{\mathrm{W}}}\left[\bar{\ell}\left(1+\gamma_{5}\right) \nu_{\ell} H^{-}+\bar{\nu}_{\ell}\left(1-\gamma_{5}\right) \ell H^{+}\right],
\end{aligned}
$$

where

$$
\alpha=\frac{\lambda_{3}}{\lambda_{1}+\lambda_{3}} \beta \lesssim \beta
$$


( $\lambda_{1}$ and $\lambda_{3}$ are coefficients in the Higgs potential: see the appendices), so $(\sin \alpha) / \beta$ $\lesssim 1$ while $\cos \alpha \simeq 1 \simeq \cos \beta$. Recall that $\beta \approx v / V$ is the (assumed small) ratio of vacuum expectation values, with $v$ setting the mass scale for light fermions and $V$ for vector bosons. Note that the interaction of $h^{0}$ is equivalent to that of the phy. sical Higgs in the standard theory if we set $\beta=\cos \alpha=1$.

For quarks,

$$
\begin{aligned}
-\mathcal{L} & =\frac{m_{\mathrm{d}} g}{2 \beta m_{\mathrm{W}}}\left[\bar{d} d \phi \sin \alpha+\bar{d} d h^{0} \cos \alpha-i \bar{d} \gamma_{5} d H^{0} \cos \beta\right] \\
& +\frac{m_{\mathrm{u}} g}{2 \beta m_{\mathrm{W}}}\left[\bar{u} u \phi \sin \alpha+\bar{u} u h^{0} \cos \alpha+i \bar{u} \gamma_{5} u H^{0} \cos \beta\right] \\
& +\frac{g \cos \theta_{\mathrm{C}}}{2 \sqrt{2} \beta m_{\mathrm{W}}}\left\{\bar{u}\left[m_{\mathrm{u}}\left(1+\gamma_{5}\right)-m_{\mathrm{d}}\left(1-\gamma_{5}\right)\right] d H^{+}\right. \\
& \left.+\bar{d}\left[-m_{\mathrm{d}}\left(1+\gamma_{5}\right)+m_{\mathrm{u}}\left(1-\gamma_{5}\right)\right] u H^{-}\right\} \\
& +\frac{g \sin \theta_{\mathrm{C}}}{2 \sqrt{2} \beta m_{\mathrm{W}}}\left\{\bar{u}\left[m_{\mathrm{u}}\left(1+\gamma_{5}\right)-m_{\mathrm{s}}\left(1-\gamma_{5}\right)\right] s H^{+}\right. \\
& \left.+\bar{s}\left[-m_{\mathrm{s}}\left(1+\gamma_{5}\right)+m_{\mathrm{u}}\left(1-\gamma_{5}\right)\right] u H^{-}\right\}
\end{aligned}
$$

with similar expressions for the interactions of other quark doublets.

This is the model we use to motivate our detailed considerations. Variations are possible, however, with more vacuum expectation values and thus more $\beta$ 's and consequently independent couplings for different fermions. To allow for this possibility in future work, and to make our phenomenological analyses as useful as possible to others, we will analyze experiments in terms of the following parameterization:

$$
\begin{aligned}
-\mathcal{L} & =\frac{g}{2 m_{\mathrm{W}}} \sum_{\ell} \frac{m_{\ell}}{\beta_{\ell}}\left[\alpha_{\ell} \ell \ell \phi+\ell \ell h^{0}+i \ell \gamma_{5} \ell H^{0}\right. \\
& \left.-\sqrt{\frac{1}{2}} \bar{\ell}\left(1+\gamma_{5}\right) \nu_{\ell} H^{-}-\sqrt{\frac{1}{2}} \bar{\nu}_{\ell}\left(1-\gamma_{5}\right) \ell H^{+}\right] \\
& +\frac{g}{2 m_{\mathrm{W}}} \sum_{q} \frac{m_{\mathrm{q}}}{\beta_{\mathrm{q}}}\left[\alpha_{\mathrm{q}} \bar{q} q \phi+\bar{q} q h^{0}+i(-1)^{\epsilon} \mathrm{q} \bar{q} \gamma_{5} q H^{0}\right] \\
& +\frac{g}{2 \sqrt{2} m_{\mathrm{W}}} \sum_{q q^{\prime}} \frac{1}{\beta_{\mathrm{qq}}}\left\{\bar{q}^{\prime}\left[-m_{\mathrm{q}}\left(1+\gamma_{5}\right)+m_{\mathrm{q}}\left(1-\gamma_{5}\right)\right] q H^{-}\right. \\
& \left.+\bar{q}\left[m_{\mathrm{q}}\left(1+\gamma_{5}\right)-m_{\mathrm{q}^{\prime}}\left(1-\gamma_{5}\right)\right] q^{\prime} H^{+}\right\},
\end{aligned}
$$

where $\epsilon_{\mathrm{q}}=0$ for charge $-\frac{1}{3}$ quarks q' and $\epsilon_{\mathrm{q}}=1$ for charge $\frac{2}{3}$ quarks $\mathrm{q}$, and $\mathrm{g}^{2} / 8 m_{\mathrm{W}}^{2}=$ 
$\sqrt{\frac{1}{2}} G_{\mathrm{F}}$. We will report results in terms of $\beta_{Q}, \beta_{\mathrm{q}}, \beta_{\mathrm{qq}}$. Note that by setting all $\beta$ 's and $\alpha$ 's equal, we regain the original model (eq. (6)). We will usually have eq. (6) in mind; however, it will be useful to see whether experiment can tell us whether, for example, $\beta_{\mathrm{e}}=\beta_{\mu}$. Details of the two-Higgs model are given in appendices $\mathrm{A}$ and $\mathrm{B}$.

We would like to make a number of remarks about the two-Higgs model. In its most general form, one may have $C P$ violation [14] and flavor-changing neutral currents [15]. We have decided not to study these features in this paper: thus for simplicity we chose a $C P$-invariant model with no flavor-changing neutral currents. The results of this paper should not depend on this choice. Even with the above simplification, the model still has several undetermined parameters (the coefficients of the Higgs potential, $\lambda_{i}$, remain free parameters). This leads to totally arbitrary Higgs masses as in the standard model. In addition, we impose a simple discrete symmetry on the Higgs sector. Then we find one Higgs mass much lighter than the others if all the $\lambda_{i}$ are of the same order and $\beta<1$. In order to simplify the phenomenology we have considered the following ansatz: $\lambda_{i}=g^{2}$ (for all $i$, where $g$ is the gauge coupling). This leads (see appendix B) to the following expressions for the Higgs masses:

$$
\begin{aligned}
& m_{\phi}=4 \sqrt{2 m_{\mathrm{W}}} \approx 450 \mathrm{GeV} / c^{2}, \\
& m_{\mathrm{H}}=2 m_{\mathrm{W}} \approx 160 \mathrm{GeV} / c^{2}, \\
& m_{\mathrm{h}}=2 \sqrt{6} m_{\mathrm{W}} \beta \approx 400 \beta \mathrm{GeV} / c^{2}
\end{aligned}
$$

Regard these as suggestive only. Note in particular that for $\beta<<1, h^{0}$ can be substantially lighter than all the other Higgs particles in the theory.

It is interesting to note that the quark masses appear explicitly, as effective couplings, in the Higgs-fermion Yukawa couplings. Consequently, it is necessary to determine what value to give to $m_{\mathrm{q}}$ when hadronic interactions are considered [16].

In processes such as deep inelastic scattering or annihilation via heavy Higgs, the renormalization-group methods suggests [17] that we use running masses evaluated at the appropriate large momentum of the process. That would imply that we should use current algebra masses such as those obtained in ref. [16]. This conclusion can be extended to other processes which admit a parton model description [18]. For example, in Drell-Yan production of dimuons, if we integrate over the transverse momentum of the massive photon, renormalization-gruup methods can be applied; the relevant large momentum here is the mass of the virtual photon. Again, one would conclude that in Drell-Yan production of dimuons by a Higgs boson, current algebra masses would be the appropriate ones to use in the Higgs-fermion coupling. The situation is much less clear in Higgs contributions to low-energy phenomena. For example, consider the charged Higgs contribution to $\beta$-decay. We will see in sect. 4 that this Higgs contribution to the scalar and pseudoscalar coupling is proportional to $m_{\mathrm{u}} \pm m_{\mathrm{d}}$, respectively. This is a low-energy process in that $\beta$-decay (e.g., neutron decay) is characterized by a mass scale equal to the neutron-proton mass difference. Hence, it would seem more appropriate to use constituent masses for the quarks. However, without a model calculation this last statement is at best an 
Table 1

Possible effects of Higgs bosons on experimental observables

\begin{tabular}{|c|c|c|c|}
\hline & Process & Observable Higgs effect & Comments \\
\hline 1. & $\begin{array}{l}(g-2)_{\mu} \\
(g-2)_{\mathrm{e}}\end{array}$ & $\begin{array}{l}\text { Too large if } \beta_{\mu}<0.015 \\
\text { Too large if } \beta_{\mathrm{e}}<5 \times 10^{-6}\end{array}$ & $\begin{array}{l}\text { Cannot rule } \\
\text { out } \beta_{\mu} \neq \beta_{\mathrm{e}}\end{array}$ \\
\hline 2. & $\begin{array}{l}\pi^{+} \rightarrow \mu^{+} \nu_{\mu} \\
\pi^{+} \rightarrow \mathrm{e}^{+} \nu_{\mathrm{e}}\end{array}$ & $\begin{array}{l}\text { No effect if } \beta_{\mathrm{e} \nu}=\beta_{\mu \nu}=\beta_{\mu} \\
\beta_{\mathrm{e}}<<\beta_{\mu \nu}<<1 \text { is rulcd out }\end{array}$ & $\begin{array}{l}\text { Pseudoscalar } \\
\text { and axial vector } \\
\text { currents give same } \\
\text { matrix element }\end{array}$ \\
\hline 3. & $\mathrm{~F}^{+}(\mathrm{c} \overline{\mathrm{s}}) \rightarrow \mathrm{l}^{+} \nu_{Q}$ & Possible & $\begin{array}{l}\text { Non-relativistic } \\
\text { quark model must be } \\
\text { trusted }\end{array}$ \\
\hline 4. & $0^{-} \rightarrow e^{+} e^{-}$ & Possible for $\eta_{\mathrm{b}}(\mathrm{b} \overline{\mathrm{b}})$ decays & $\begin{array}{l}\text { Requires a light pseudo } \\
\text { scalar Higgs Boson }\end{array}$ \\
\hline
\end{tabular}

5. $0^{+} \rightarrow \ell^{+} \ell^{-}$

6. $\Upsilon \rightarrow h^{0} \gamma$

7. $\mathrm{e}^{+} \mathrm{e}^{-} \rightarrow \mu^{+} \mu^{-}$ $\left(\mathrm{q}^{\bar{q}} \rightarrow \ell^{+} \ell^{-}\right)$
8. Drell-Yan

Possible for $\chi(\mathrm{cc})$ and $\mathrm{x}_{\mathrm{b}}(\mathrm{b})$ decays. The decay $\mathrm{X}_{\mathrm{b}} \rightarrow \tau^{+} \tau^{-}$could put strong limits on $\beta$

Will put strong limits on $\beta$ if $m_{\mathrm{h}}<m_{\Upsilon}$.

No chance unless $\beta_{\mathrm{e}} \ll \beta_{\mu} \ll 1$

Possible if one can examine $\tau^{+} \tau^{-}$final states, if any $\beta<0.01$ or if $m_{\mathrm{q}}$ greater than current quark mass. $\mathrm{K}^{+} \mathrm{N} \rightarrow \mu^{+} \mu^{-} \mathrm{X}$ is best place to search for Higgs
9. $\quad \mathrm{eN} \rightarrow \mathrm{eX}$ $\mu \mathrm{N} \rightarrow \mu \mathrm{X}\}$

10. $\mu^{-} \rightarrow \mathrm{e}^{-} \nu_{\mu} \bar{\nu}_{\mathrm{e}}$

Weak limits on $\beta_{\mathrm{e} \nu}, \beta_{\mu \nu}$

Weak limits on $\beta_{\mathrm{e} \nu}, \beta_{\mathrm{ud}}$

No chance; however $\beta_{\mathbf{f}}=m_{\mathfrak{f}} / 2 m_{\mathrm{W}}$ is ruled out

11. Beta decay

12. $\nu \mathrm{N} \rightarrow \mu^{-} \mathrm{X}$
If $m_{\mathrm{h}} \approx m_{\mathrm{Xb}}$ then this will be observable even in the standard model. Forbidden by CVC for vector current.

Possible even if $\beta=1$

Careful experimental study worth while, both for rate and forward-backward asymmetry
Experimental limits are fairly weak

Perhaps one could get useful limits by studying data in small $x$ region: Higgs and QCD scaling violations contribute to same term and cancel

13. Parity violation Results of SLAC $\overrightarrow{e d} \rightarrow \mathrm{eX}$ in electron- experiment $[10]$ rules out nucleon interaction significant Higgs effects 
Table 1 (continued)

\begin{tabular}{lll}
\hline Process & Observable Higgs effect & Comments \\
\hline $\begin{array}{l}\mathrm{K}_{\mathrm{L}}^{0}-\mathrm{K}_{\mathrm{S}}^{0} \text { mass } \\
\text { difference and } \\
\text { flavor changing } \\
\text { neutral currents }\end{array}$ & $\begin{array}{l}\text { For } m_{\mathrm{H}} \geq m_{\mathrm{W}} \text {, the Higgs-fermion } \\
\text { coupling could be as large as } 30 \%\end{array}$ & of $g[44]$
\end{tabular}

The existence of the following Higgs particles is assumed: a light scalar $\mathrm{h}^{0}\left(m_{\mathrm{h}}<m_{\mathrm{W}}\right.$ ), a heavy pseudoscalar $\mathrm{H}^{0}\left(m_{\mathrm{H}}>m_{\mathrm{W}}\right)$ and heavy charged $\mathrm{H}^{ \pm}$(we assume $m_{\mathrm{H}^{+}}=m_{\mathrm{H}^{0}}$ for convenience). The Higgs-fermion interaction is enhanced by a factor $\beta^{-1}$ over that of the Weinberg-Salam model. We allow for the possibility that $\beta$ depends on the fermions involved.

order of magnitude guess. We would like to point to a calculation done in ref. [19] where the authors attempt to calculate the neutral Higgs-nucleon coupling. Their result is that the Higgs-nucleon coupling (in low-energy transitions involving $u$ and $\mathrm{d}$ quarks) is proportional to a mass substantially larger than the current quark mass (though probably less than a constituent quark mass). We conclude that for phenomenological purposes, we can treat $m_{\mathrm{q}}$ as a current algebra mass for processes involving large mass scales and constituent quark mass for low-energy phenomena.

There are clearly many consequences of our viewpoint for production of Higgs particles. On the assumption that the heavy Higgs are very heavy, say $m_{\mathrm{H}} \gtrsim m_{\mathrm{W}}$, they would mainly be seen in $\mathrm{e}^{+} \mathrm{e}^{-}$collisions or pair produced, as with $\mathrm{W}^{ \pm}, \mathrm{Z}$. The production of $h^{0}$ should be considerably easier since it is lighter, perhaps in the few $\mathrm{GeV}$ range. The coupling of $\mathrm{h}^{0}$ to electrons is still proportional to $m_{\mathrm{e}}$, hence the production of $\mathrm{h}^{0}$ in $\mathrm{e}^{+} \mathrm{e}^{-}$would be suppressed even in the case of an enhanced fermion-Higgs coupling. In the present paper, we will not consider in any detail production mechanisms and direct observation of the Higgs particles. We will concentrate on determining the restrictions from low-energy phenomenology on the allowed ranges of $\beta_{\mathrm{q}}$ and $\beta_{\ell}$ since this can be done accurately. In a future paper we will study the production and detection questions if the numerical results still seem to deserve consideration.

The rest of the paper is organized as follows. In sect. 2, we will present the major experimental constraints on the parameters of our model. Using the Higgs masses which resemble those of eq. (8), we find that $g-2$ of the muon leads to a lower limit on $\beta$. By studying $\pi^{ \pm}$decay, we can obtain limits on the possible inequality $\beta_{\mathrm{e}} \neq \beta_{\mu}$. All further experimental results presently available do not constrain the model significantly. There are many interesting tests which can be done in the near future. In sects. 3,4 we discuss ways in which to observe effects of the neutral and charged Higgs bosons. In table 1 , we present in summary from the various processes which could show effects of the Higgs bosons. Finally, in sect. 5, we give our conclusions. The details of the two-Higgs model and a discussion of amplitudes for fermion- 
antifermion scattering (where one might look for Higgs effects) are given in the appendices.

\section{Major experimental constraints}

\section{1. $(g-2)$ of the muon and electron}

From the useful summary paper of Leveille [20], we can read off the contributions of our Higgs particles to $(g-2)$ of the muon. (See fig. 1.) For $h^{0}$ and $H^{0}$, we have (using his eq. (11)):

$$
a_{\mu}^{\mathrm{h}^{0}}=\frac{C_{\mathrm{s}}^{2}}{8 \pi^{2}} \int_{0}^{1} \mathrm{~d} x\left(2 x^{2}-x^{3}\right) /\left(x^{2}+\Lambda_{\mathrm{h}}(1-x)\right),
$$

where $a_{\mu} \equiv \frac{1}{2}(g-2)$ of the $\mu^{--}, C_{\mathrm{s}} \equiv g m_{\mu} / 2 \beta_{\mu} m_{\mathrm{W}}$ and $\Lambda_{\mathrm{h}}=m_{\mathrm{h}}^{2} / m_{\mu}^{2}$, and

$$
a_{\mu}^{\mathrm{H}^{0}}=\frac{-C_{\mathrm{p}}^{2}}{8 \pi^{2}} \int_{0}^{1} \mathrm{~d} x x^{3} /\left(x^{2}+\Lambda_{\mathrm{H}}(1-x)\right),
$$

where $C_{\mathrm{p}} \equiv C_{\mathrm{s}}$ in our model and $\Lambda_{\mathrm{H}} \equiv m_{\mathrm{H}}^{2} / m_{\mu}^{2}$. For the charged Higgs $\mathrm{H}^{\ddagger}$, we obtain (using his eq. (10)):

$$
a_{\mu}^{\mathrm{H}^{ \pm}} \approx \frac{-C_{\mathrm{s}}^{2}}{8 \pi^{2}} \int_{0}^{1} \mathrm{~d} x x(1-x) /\left(x+\Lambda_{\mathrm{H}}\right) .
$$

Note that $\Lambda$ is large for Higgs masses in the range which we consider. Therefore, we find that the total Higgs contribution is approximately:

$$
a_{\mu}^{\text {Higgs }}=\frac{C_{\mathrm{s}}^{2}}{8 \pi^{2}}\left[\left(\frac{\ln \Lambda_{\mathrm{h}}-\frac{7}{6}}{\Lambda_{\mathrm{h}}}\right)-\left(\frac{\ln \Lambda_{\mathrm{H}}-2}{\Lambda_{\mathrm{H}}}\right)\right] .
$$

Using the masses given by eq. (8), we can drop the second term above. The current experimental data and theory allow a contribution from other sources; if all of the discrepancy were taken up here ${ }^{\star}$, we would have

$$
a_{\mu}^{\text {Higgs }} \lesssim 2 \times 10^{-8}
$$

From eq. (12), we find (all masses in GeV units)

$$
a_{\mu}^{\text {Higgs }} \approx 2.6 \times 10^{-11}\left(\ln \Lambda_{\mathrm{h}}-\frac{7}{6}\right) / \beta_{\mu}^{2} m_{\mathrm{h}}^{2} .
$$

We can get a limit on $\beta_{\mu}$ by choosing a mass for $m_{\mathrm{h}}$. For example, if we use (cf.,

* The $\mathrm{Z}^{0}$ and $\mathrm{W}^{ \pm}$contributions to $a_{\mu}$ are [51] only about $0.2 \times 10^{-8}$, hence we may safely neglect them here. 




(a)

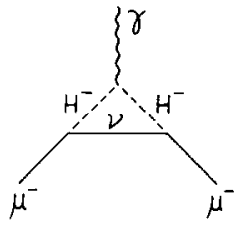

(b)

Fig. 1. The Higgs contributions to $(g-2)$ of the muon: (a) neutral Higgs contribution and (b) charged Higgs contribution.

eq. (8)), $m_{\mathrm{h}} \approx 400 \beta_{\mu} \mathrm{GeV}$ we find

$$
\beta_{\mu} \gtrsim 0.015
$$

(which corresponds to $m_{\mathrm{h}} \gtrsim 6 \mathrm{GeV} / c^{2}$ ). Alternatively, we may obtain a limit on the product $\beta_{\mu} m_{\mathrm{h}}$. In fact, this limit will be less sensitive to the actual value of the Higgs mass since it depends only on $\ln \Lambda_{\mathrm{h}}$. Thus, if $\ln \Lambda_{\mathrm{h}} \approx 10$, we obtain $\beta_{\mu} m_{\mathrm{h}} \geq 0.1$. It should be noted that eq. (15) is based on an arbitrary choice for the value of $m_{\mathbf{h}}$. If we leave $m_{\mathrm{h}}$ as a free parameter, then one can change the limits on $\beta$ by an order of magnitude by a corresponding change in the value of $m_{\mathrm{h}}$. Furthermore, if $m_{\mathrm{h}}$ is of the same order as $m_{\mathrm{H}}$, then it is possible to have the contributions to $a_{\mu}^{\mathrm{Higgs}}$ cancel out (see eq. (12)). In addition, if this model is generalized to include an axion (see further discussion in sect. 5), then with two Higgs and $\beta<<1$ it can happen that the axion mass is similar to the $h^{0}$ mass [21]. Since the axion is pseudoscalar, the axion and $h^{0}$ contributions to $(g-2)_{\mu}$ cancel, and perhaps could significantly lower the above limit.

In this paper we will use eq. (15) as a guide; we will, however, keep the remarks above in mind. In particular, note that if all Higgs masses are very large (say, larger then $m_{\mathrm{W}}$ ) then $a_{\mu}^{\mathrm{Higgs}}$ places a very mild restriction on $\beta_{\mu}$. For example, $\beta_{\mu}=m_{\mu} / 2 m_{\mathrm{W}}$ $=7 \times 10^{-4}$ could not be ruled out. Such large Higgs masses would totally suppress any Higgs contributions to low-energy phenomenology. If such a small $\beta_{\mu}$ existed there could be observable effects in very high-energy reactions $[5,7]$.

Turning to the electron $(g-2)$, we use the experimental limit [51]

$$
a_{\mathrm{e}}^{\text {Higgs }} \lesssim 3 \times 10^{-10} .
$$

The theoretical Higgs contribution for the electron gets smaller than that of the muon by roughly a factor $\left(m_{\mathrm{e}} / m_{\mu}\right)^{4}$. Since the experimental limit for the electron is only about 100 times better than that for the muon, the resulting limit for $\beta_{\mathrm{e}}$ is not as strong as compared with $\beta_{\mu}$. If we take $m_{\mathrm{h}} \geq 6 \mathrm{GeV} / c^{2}$ as before, we find

$$
\beta_{\mathrm{e}} \gtrsim 4.8 \times 10^{-6} \text {. }
$$

Therefore, $(g-2)$ of the electron does not give us any useful restriction.

The $(g-2)_{\tau}$ will have a large contribution from the Higgs. Even in the standard Weinberg-Salam model the Higgs contribution is greater than the hadronic contri- 


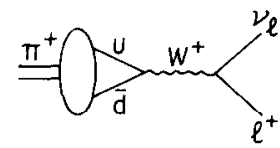

(a)

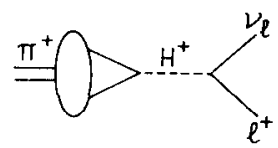

(b)

Fig. 2. Quark model picture of $\pi^{+} \rightarrow \ell^{+} p_{\ell}\left(\ell^{+}=\mathrm{e}^{+}\right.$or $\mu^{+}$): (a) $\mathrm{W}^{+}$contribution (this is the standard weak interaction description) and (b) $\mathrm{H}^{+}$contribution.

bution if $m_{\ell} \geqslant 4 \mathrm{GeV}[22]$. In our model the effect is even larger, $\Lambda_{\mathrm{h}} \approx 9$ and we find $a_{\tau}^{\text {Higgs }} \approx 7 \times 10^{-8} / \beta_{\tau}^{2}$. For $\beta \approx .015, a_{\tau}^{\text {Higgs }} \approx 3 \times 10^{-4}$ which is about a third the anomoly from QED.

\subsection{Leptonic decays of $\pi^{ \pm}$and $K^{ \pm}$}

In the two-Higgs model discussed in appendix $\mathrm{B}$ there is only one parameter $\beta$; that is, $\beta_{\mu}=\beta_{\mathrm{e}}$. One can imagine a more complicated theory in which $\beta_{\mu} \neq \beta_{\mathrm{e}}$. The results of $(g-2)$ give us no information on this possibility. One might expect to be able to learn something new by looking at the $\mu$-e universality tests. Consider the case of $\pi^{ \pm}$decay into $\mu^{ \pm} \nu$ or $\mathrm{e}^{ \pm} \nu$. In addition to the $\mathrm{W}$ contribution (fig. $2 \mathrm{a}$ ), one must also include the effects of the charged Higgs (fig. 2b). One might be able to constrain the Higgs coupling using the experimental results for $\Gamma(\pi \rightarrow \mathrm{e} \nu) / \Gamma(\pi \rightarrow \mu \nu)$; in fact, no strong constraint arises. Recall that apart from phase space, $\Gamma(\pi \rightarrow \mathrm{e} \nu)$ / $\Gamma(\pi \rightarrow \mu \nu)$ is proportional to $m_{\mathrm{e}}^{2} / m_{\mu}^{2}$ for a $\mathrm{V}$, A contribution but independent of the lepton masses for a scalar contribution. For a Higgs coupling proportional to lepton masses, it is anusing that the Higgs scalar current also gives a contribution proportional to $m_{\ell}^{2}$, so the above ratio is unchanged in the case of $\beta_{\mathrm{e}}=\beta_{\mu}$. Therefore, one can only really constrain the possibility that $\beta_{\mathrm{e}} \neq \beta_{\mu}$ in such a test ; such a constraint can exist only if the Higgs contribution is appreciable.

We now turn to details of the calculation. We imagine the $\pi$-meson as a bound state of quarks (ud) and perform the standard [23] non-relativistic calculation. First, let us consider the $\mathrm{W}$ contribution alone. One finds [24]

$$
\Gamma\left(\pi^{+} \rightarrow \ell^{+} \nu_{\ell}\right)=\frac{3 G_{\Gamma}^{2} \cos ^{2} \theta_{\mathrm{C}} m_{\ell}^{2}\left(1-m_{\ell}^{2} / m_{\pi}^{2}\right)^{2}\left|R_{\mathrm{s}}(0)\right|^{2}}{8 \pi^{2}},
$$

where $R_{\mathrm{s}}(0)$ is the value of the s-wave radial wave function at the origin. (For the decay of $\mathbf{K}^{+}$, replace $\cos \theta_{\mathbf{C}}$ with $\sin \theta_{\mathrm{C}}$ and $m_{\pi}$ with $m_{\mathbf{K}}$ ). If we use Jackson's [24] fit to the leptonic decay of vector mesons (in which he finds $\left|R_{\mathrm{s}}(0)\right|^{2} \propto M^{1.89}$ $\mathrm{GeV}^{3}$ ), we obtain results for $\pi$ and $\mathrm{K}$ decay that are within a factor of two of the experimental results ${ }^{\star}$. Given the relativistic nature of the ud system in the pion and

\footnotetext{
* One could regard the charged $\pi$ decay result as coincidental. For example, if one computes $\pi^{0} \rightarrow 2 \gamma$ by similar methods (analogous to the $\eta_{\mathrm{C}} \rightarrow 2 \gamma$ calculation), one obtains a result 300 times larger than the experimental data.
} 
the uncertainty of $R_{\mathrm{s}}(0)$, this result is certainly satisfactory.

When we include the charged Higgs contribution the result is

$$
\Gamma\left(\pi^{+} \rightarrow \ell^{+} \nu_{\ell}\right)=\Gamma_{0}\left[1-\frac{m_{\pi}\left(m_{\mathrm{u}}+m_{\mathrm{d}}\right)}{\beta_{\ell \nu} \beta_{\mathrm{ud}}\left(m_{\mathrm{H}}^{2}-m_{\pi}^{2}\right)}\right]^{2},
$$

where $\Gamma_{0}$ is the width calculated in eq. (18). As promised, if $\beta_{\mathrm{e} \nu}=\beta_{\mu \nu}$, then $\Gamma\left(\pi^{+} \rightarrow \mathrm{e}^{+} \nu\right) / \Gamma\left(\pi^{+} \rightarrow \mu^{+} \nu\right)$ remains unchanged. Note that the absolute rate is modified in eq. (19), but we can just as well use the expression in brackets to redefine $R_{\mathrm{s}}(0)$. This effectively renormalizes $f_{\pi}^{2}=3\left|R_{\mathrm{s}}(0)\right|^{2} / \pi m_{\pi}$. Unless $R_{\mathrm{s}}(0)$ or equivalently $f_{\pi}$ is known accurately from an independent source, the absolute rate tells us nothing about the Higgs contribution.

Suppose $\beta_{\mathrm{e} \nu} \neq \beta_{\mu \nu}$. Then, assuming the second term in eq. (19) is small, we have (for $m_{\pi}<<m_{\mathrm{H}}$ )

$$
\frac{\Gamma\left(\pi^{+} \rightarrow \mathrm{e}^{+} \nu\right)}{\Gamma\left(\pi^{+} \rightarrow \mu^{+} \nu\right)}=\frac{\Gamma_{0}\left(\pi^{+} \rightarrow \mathrm{e}^{+} \nu\right)}{\Gamma_{0}\left(\pi^{+} \rightarrow \mu^{+} \nu\right)}\left[1-\frac{2 m_{\pi}\left(m_{\mathrm{u}}+m_{\mathrm{d}}\right)}{\beta_{\mathrm{ud}} m_{\mathrm{H}}^{2}}\left(\frac{1}{\beta_{\mathrm{e} \nu}}-\frac{1}{\beta_{\mu \nu}}\right)\right] .
$$

As discussed in sect. 1, we use current algebra masses for the quarks [16] $\left(m_{\mathrm{u}}+\right.$ $m_{\mathrm{d}} \approx 10 \mathrm{MeV} / c^{2}$ ). Then, to satisfy $\mu$-e universality,

$$
\frac{2 m_{\pi}\left(m_{\mathrm{u}}+m_{\mathrm{d}}\right)}{\beta_{\mathrm{ud}} m_{\mathrm{H}}^{2}}\left(\frac{1}{\beta_{\mathrm{e} \nu}}-\frac{1}{\beta_{\mu \nu}}\right)<<1 .
$$

If we take $m_{\mathrm{H}} \approx 160 \mathrm{GeV} / c^{2}$ (from our estimate given in eq. (8)), then one could get constraints on the possibility that $\beta_{\mathrm{e} \nu}<<\beta_{\mu \nu}<<1$ (which would be the case if $\left.\beta_{\ell \nu}=m_{\ell} / 2 m_{\mathrm{W}}\right)$. For example, if we took $\beta_{\mathrm{ud}}=\beta_{\mu \nu} \approx 10^{-2}$ then we would find $\beta_{\mathrm{e} \nu}>>10^{-5}$. Note that if the charged Higgs mass were much lighter, then the above test would definitely rule out $\beta_{\mathrm{e} \nu} \neq \beta_{\mu \nu}$.

As a final remark, one can also use the above results for analyzing the decays of other charged pseudoscalars. For example, for $\mathrm{K}^{ \pm} \rightarrow \ell^{ \pm} \nu_{\ell}$, one would replace $m_{\pi}$ in the previous four equations with $m_{\mathrm{K}}$ and (assuming $\beta_{\mathrm{ud}}=\beta_{\mathrm{us}}=\beta_{\mathrm{ds}}$ ) obtain slightly stronger limits on $\beta_{\ell \nu}$. A potentially interesting decay to study in the future is leptonic decays of the charmed $\mathrm{F}^{+}$meson. These decays are not Cabibbo suppressed, so that eventually one might have enough events to observe them. In addition, one might have more trust in the value of $R_{\mathrm{s}}(0)$ at the $\mathrm{F}$ mass; in that case, comparison of experiment results with the absolute rate given by eq. (19) could be useful.

\subsection{Summary of results}

Let us briefly review the constraints we have obtained thus far. If we accept, qualitatively, the Higgs mass spectrum suggested by eq. (8), we find from $(g-2)$ of the muon and from $\pi$ (or $\mathrm{K}$ ) decay that it is likely that $\beta_{\mu \nu}=\beta_{\mathrm{e} \nu}$ and $\beta \geq 10^{-2}$. If we relax the condition on the Higgs masses by making them heavier, then clearly one can end up with no useful restrictions on $\beta$. It may be worth keeping in mind that bizarre situations such as $\beta_{\mathrm{e}} \neq \beta_{\mu}$ but $\beta_{\mathrm{e} \nu}=\beta_{\mu \nu}$ have not not been ruled out in the above analysis. 
However, if we now proceed to look in other areas of particle physics where Higgs effects can show up, we discover two results. First, current experimental data can not put bounds on our model more restrictive than the ones we have just obtained. Second, there are a number of experiments feasible in the near future which can help put constraints on our model. We now turn to a discussion of these points.

\section{Finding effects of the neutral Higgs bosons}

In our nudel we have both a scalar and a pseudoscalar Higgs boson. Thus, one can have Higgs contributing to the decays of both $0^{+}$and $0^{-}$mesons. Furthermore, by investigating neutral current processes, one can hopefully determine any presence of a scalar or pseudoscalar current. In this section we investigate some examples when one might hope to see evidence for the neutral Higgs boson.

\subsection{Decay of neutral pseudoscalar mesons}

The decays $\pi^{0} \rightarrow \mathrm{e}^{+} \mathrm{e}^{-}$and $\eta^{0} \rightarrow \mu^{+} \mu^{-}$are rare; they must proceed electromagnetically in second order (see fig. 3a). In addition, such a process may occur via the weak neutral current ( $Z$ exchange) or by exchange of a pseudoscalar Higgs particle $\mathrm{H}^{0}$ (see figs. $3 \mathrm{~b}, \mathrm{c}$ ). If the decay rate $\mathrm{P} \rightarrow \gamma \gamma(\mathrm{P}=$ pseudoscalar meson) is known experimentally, the imaginary part of the QED amplitude for $\mathrm{P} \rightarrow \ell^{+} \ell^{-}$can be deternined using unitarity [25], hence a lower bound for $\Gamma\left(\mathrm{P} \rightarrow \ell^{+} \ell^{-}\right) / \Gamma(\mathrm{P} \rightarrow \gamma \gamma)$ can be rigorously obtained. Unfortunately, the real part of the QED amplitude is model dependent and usually depends on an unknown parameter. For "reasonable" values of that parameter one usually finds that the real part of the QED amplitude is approximately equal to the imaginary part. However, the experimental results for $\pi^{0} \rightarrow \mathrm{e}^{+} \mathrm{e}^{-}$and $\mu^{+} \mu^{-}[26]$ are typically a factor of five (with large errors) above the unitarity bound. Therefore there is room for other contributions. This alone would not constitute a proof of the existence of Higgs exchange, although it could provide

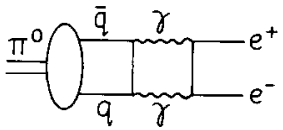

(a)

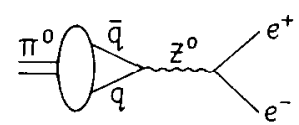

(b)

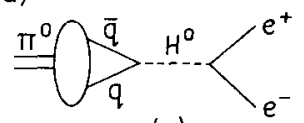

(c)

Fig. 3. Contributions to $\pi^{0} \rightarrow \mathrm{e}^{+} \mathrm{e}^{-\cdots}$ : (a) electromagnetic contribution (related by unitarity to $\pi^{0} \rightarrow \gamma \gamma$ ); (b) neutral current $\mathrm{Z}^{0}$ contribution; (c) neutral Higgs $\mathrm{H}^{0}$ contribution: note that $\mathrm{H}^{0}$ is a pscudoscalar boson in our model. 
some interesting limits on the Higgs-fermion coupling.

We now present our calculation. For completeness we record the unitarity bound [25] for $\mathrm{P} \rightarrow \ell^{+} \ell^{-}$:

$$
\frac{\Gamma\left(\mathrm{P} \rightarrow \ell^{+} \ell^{-}\right)}{\Gamma(\mathrm{P} \rightarrow \gamma \gamma)} \geqslant \frac{2 \alpha^{2} m_{\ell}^{2}}{M^{2}}\left(1-\frac{4 m_{\ell}^{2}}{M^{2}}\right)^{-1 / 2}\left[\ln \left(\frac{M+\sqrt{M^{2}-4 m_{\ell}^{2}}}{2 m_{\ell}}\right)\right]^{2},
$$

where $M$ is the mass of $\mathrm{P}$. To calculate the $\mathrm{Z}$ and $\mathrm{H}^{0}$ contributions, we again use nonrelativistic quark model techniques. The result:

$$
\Gamma\left(\mathrm{P} \rightarrow \ell^{+} \ell^{-}\right)=C\left[g_{\mathrm{A}} g_{\mathrm{A}^{\prime}}+\frac{m_{\mathrm{q}} M}{\beta_{\ell} \beta_{\mathrm{q}}\left(m_{\mathrm{H}}^{2}-M^{2}\right)}\right]^{2}\left|R_{\mathrm{s}}(0)\right|^{2},
$$

where $C \equiv 3 G_{\mathrm{F}}^{2} m_{\ell}^{2}\left(1-4 m_{\ell}^{2} / M^{2}\right)^{1 / 2} / 4 \pi^{2}$ and $g_{\mathrm{A}}, g_{\mathrm{A}^{\prime}}=-\sqrt{2} T_{3}$ for an incoming (outgoing) fermion of weak isospin $T_{3}$. Eq. (23) holds for each q $\bar{q}$ in P; one must then correctly put in the appropriate SU(3) wave function of $P$.

Let us consider the following decays: $\pi^{0} \rightarrow \mathrm{e}^{+} \mathrm{e}^{-}, \eta, \eta^{\prime}$, and $\eta_{\mathrm{c}} \rightarrow \mu^{+} \mu^{--}$, and $\eta_{\mathrm{b}} \rightarrow \tau^{+} \tau^{-}$where $\eta_{\mathrm{c}}\left(\eta_{\mathrm{b}}\right)$ is the $0^{-+} \overline{\mathrm{c}}(\mathrm{b} \overline{\mathrm{b}})$ ground state. To be definite, we identify $\chi(2.83)$ with $\eta_{\mathrm{c}}$ and choose the $\eta_{\mathrm{b}}$ mass to be $9 \mathrm{GeV} / \mathrm{c}^{2}$. We now substitute the appropriate numbers into eq. (23). For definiteness, let us choose $\beta_{\ell}=\beta_{\mathrm{q}}=0.015$ and $m_{\mathrm{H}}=160 \mathrm{GeV} / c^{2}$ : we immediately discover that the $\mathrm{Z}$ contribution dominates the Higgs contribution for the light pseudoscalar decays. This is due to the small size of the quark mass and the large value we use for the Higgs mass. Even if we stretched our parameters to the limit by using, for example, $\beta_{\mathrm{f}}=m_{\mathrm{f}} / 2 m_{\mathrm{W}}$ and $m_{\mathrm{H}}=$ $16 \mathrm{GeV} / c^{2}$, we would not obtain an observable Higgs contribution for the light pseudoscalar decays. In the latter case, the Higgs contribution would indeed dominate the $\mathrm{Z}$ contribution but would still be at least an order of magnitude (or more) smaller than the unitarity limit given by eq. (22).

For the heavier pseudoscalars, $\left(\eta_{\mathrm{c}}, \eta_{\mathrm{b}}\right)$, a rate at the level of the unitarity limit is experimentally unobservable. One needs a decay many orders of magnitude larger in order that the Higgs contribution be detectable. If we choose $\beta_{\mathrm{q}}=\beta_{\ell}=0.015$ and $m_{\mathrm{H}}=160 \mathrm{GeV} / c^{2}$, we find that the Higgs contribution is too small to be seen. However, consider what happens if $m_{\mathrm{II}}$ is much lighter than we have imagined. For example, in the case of $\eta_{\mathrm{b}}$ decay, choose $m_{\mathrm{b}}=4.5 \mathrm{GeV} / c^{2}, \beta=0.015$ and $m_{\mathrm{H}}=16$ $\mathrm{GeV} / c^{2}$. One then finds that the Higgs contributions $\Gamma\left(\eta_{\mathrm{b}} \rightarrow \tau^{+} \tau^{-}\right) \approx 130 \mathrm{keV}$. This is a reasonable rate to see a signal assuming one could be sure one was producing an $\eta_{\mathrm{b}}$ (e.g., $\mathrm{e}^{+} \mathrm{e}^{-} \rightarrow \Upsilon \rightarrow \eta_{\mathrm{b}} \gamma \rightarrow \tau^{+} \tau^{-} \gamma$ which would be identified by searching for $\mathrm{e}^{ \pm} \mu^{\mp} \gamma$ events). Note that even with such a light $\mathrm{H}^{0}$, the $\eta_{\mathrm{c}} \rightarrow \mu^{+} \mu^{-}$decay would be unobservable. In order that the $\mathrm{H}^{0}$ contribution be appreciable for the $\eta_{\mathrm{c}}$ decay, one would need substantially smaller values for $\beta$.

\subsection{Decay of neutral scalar mesons}

The best understood scalar meson is the $\chi(3415)$, the $0^{++}$state in charmonium. Perhaps in the next few years the andogous state in the $b \bar{b}$ system (which we 
denote by $\chi_{b}$ ) will also be well studied. This meson has the quantum numbers of the light Higgs meson $h^{0}$ in our model and therefore could be an ideal place to search for Higgs effects. In fact, weak and electromagnetic decays into $\ell^{+} \ell^{-}$are forbidden in the decay of scalar mesons due to the conserved vector current. Hence, observation of a $\ell^{+} \ell^{-}$decay of a scalar meson could be proof of a scalar current in general and a Higgs meson in particular.

Consider then the decay of the $\chi$ into $\ell^{+} \ell^{-}$(via an $\mathrm{h}^{0}$ intermediate state). Again, we use the non-relativistic quark model; the result is

$$
\Gamma\left(\chi \rightarrow \ell^{+} \ell^{-}\right)=\frac{27 G_{\mathrm{F}}^{2} m_{\ell}^{2} M^{2}\left(1-4 m_{\ell}^{2} / M^{2}\right)^{3 / 2}\left|R_{\mathrm{p}}^{\prime}(0)\right|^{2}}{4 \pi^{2} \beta_{\ell}^{2} \beta_{\mathrm{q}}^{2}\left[\left(m_{\mathrm{h}}^{2}-M^{2}\right)^{2}+m_{\mathrm{h}}^{2} \Gamma_{\mathrm{h}}^{2}\right]},
$$

where $M$ is the mass of the $\chi$ and $R_{\mathrm{p}}^{\prime}(0)$ is the derivative of the radial p-state wave function at the origin. Note that we have allowed for the fact that $m_{\mathbf{h}}$ might be close to $M$ (using cq. (8), $m_{\mathrm{h}} \gtrsim 6 \mathrm{GeV} / c^{2}$ ) by inserting a Breit-Wigner denominator.

In order to see how large this can be, let us normalize the width to [24] $\Gamma(\chi \rightarrow g g)=96 \alpha_{\mathrm{s}}^{2}\left|R_{\mathrm{p}}^{\prime}(0)\right|^{2} / M^{4} \approx 1-3 \mathrm{MeV}$ for $\mathrm{c} \overline{\mathrm{c}}$ and $\mathrm{b} \overline{\mathrm{b}}$ systems. (In these analyses, $\alpha_{\mathrm{s}}=0.19$ for $\mathrm{c} \overline{\mathrm{c}}$ and $\alpha_{\mathrm{s}}=0.15$ for $\mathrm{b} \overline{\mathrm{b}}$ ). We then find

$$
\frac{\Gamma\left(\chi \rightarrow \ell^{+} \ell^{-}\right)}{\Gamma(\chi \rightarrow \mathrm{gg})}=\frac{9 G_{\mathrm{F}}^{2} m_{\ell}^{2} M^{6}\left(1-4 m_{\ell}^{2} / M^{2}\right)^{3 / 2}}{128 \pi^{2} \alpha_{\mathrm{s}}^{2} \beta_{\ell}^{2} \beta_{\mathrm{q}}^{2}\left[\left(m_{\mathrm{h}}^{2}-M^{2}\right)^{2}+m_{\mathrm{h}}^{2} \Gamma_{\mathrm{h}}^{2}\right]} .
$$

First, let us examine the unlikely but amusing situation that $M$ is very close to the Higgs mass. For example, a Higgs mass of $10 \mathrm{GeV} / c^{2}$ could be very close to the mass of the $\chi_{b}$. We then use eq. (25) and consider the decay $\chi_{b} \rightarrow \tau^{+} \tau^{-}$. Since $h$ couples to fermions with coupling proportional to the fermion mass, we approximate $\Gamma_{\mathrm{h}}=\Gamma(\mathrm{h} \rightarrow \overrightarrow{\mathrm{c}} \overline{\mathrm{c}})+\Gamma\left(\mathrm{h} \rightarrow \tau^{+} \tau^{-}\right)$(remember that we have in mind that $m_{\mathrm{h}} \approx$ $m_{\chi_{\mathrm{b}}}$ which is below $\mathrm{b} \overline{\mathrm{b}}$ threshold). Then we find

$$
\Gamma_{\mathrm{h}}=\frac{G_{\mathrm{F}} m_{\mathrm{h}}\left(3 m_{\mathrm{C}}^{2}+m_{\tau}^{2}\right)}{4 \sqrt{2} \pi \beta^{2}} .
$$

Thus, we find $\Gamma_{\mathrm{h}} / m_{\mathrm{h}} \approx 6 \times 10^{-6} / \beta^{2}$.

If $\left|m_{\mathrm{h}}-M\right|<m_{\mathrm{h}} \Gamma_{\mathrm{h}}$, then one would expect mixing between the $\chi_{\mathrm{b}}$ and the Higgs boson. Even in the more likely case that $\left|m_{\mathrm{h}}-M\right| \gg m_{\mathrm{h}} \Gamma_{\mathrm{h}}$, there could be a reasonable chance for an observable signal. The reason is that the signal is very clean from background if one looks for the $\tau^{+} \tau^{-}$decay. One would sit on the $\Upsilon^{\prime}$ and watch the decay $\Upsilon^{\prime} \rightarrow \gamma \chi_{b} \rightarrow \gamma \tau^{+} \tau^{-}$which would be identified by $\gamma \mathrm{e}^{ \pm} \mu^{+}$events where the $\gamma$ is monochromatic. As a numerical example, consider the case where $\beta=0.015$ and $m_{\mathrm{h}}=6 \mathrm{GeV} / c^{2}$. We then find that $\Gamma\left(\chi_{\mathrm{b}} \rightarrow \tau^{+} \tau^{-}\right) \approx 1 \mathrm{MeV}$ which would be quite an observable width. If on the other hand $\beta=1$, we would need a much more fortuitous circumstance of $m_{\mathrm{h}}$ being very near the mass of the $\chi_{\mathrm{b}}$ in order that the $\chi_{b} \rightarrow \tau^{+} \tau^{-}$decay be observable.

Second, let us examine the Higgs contribution to $\chi(3415)$ decay. Using the same numbers as before $\left(\beta=0.015\right.$ and $\left.m_{\mathrm{h}}=6 \mathrm{GeV} / c^{2}\right)$ we find that $\Gamma\left(\chi \rightarrow \mu^{+} \mu^{-}\right) \approx$ 
$0.02 \mathrm{keV}$. Note in particular the factor of $\beta^{-4}$ enhancement in eq. (25) without which this reaction would be unobservable. Perhaps one could put useful limits on $\beta m_{\mathrm{h}}$ with a high-statistics study of $\psi^{\prime}$ decays.

\subsection{Decay of neutral vector mesons}

For completeness, we mention a process proposed by Wilczek [4]: production of $\mathrm{h}^{0}$ (or $\mathrm{H}^{0}$ ) by decay of a neutral vector meson $\mathrm{q} \overline{\mathrm{q}}$ state: $\mathrm{V}^{0} \rightarrow \mathrm{H}^{0} \gamma$. Even in the Weinberg-Salam model, there is a chance this decay will be observed if the Higgs mass is light enough. If $\beta \neq 1$, then this method becomes an excellent one for producing the Higgs. For a scalar or pseudoscalar Higgs, the result is the same:

$$
\frac{\Gamma\left(\mathrm{V}^{0} \rightarrow \mathrm{h}^{0} \gamma\right)}{\Gamma\left(\mathrm{V}^{0} \rightarrow \mu^{+} \mu^{-}\right)}=\frac{G_{\mathrm{F}}\left(M^{2}-m_{\mathrm{h}}^{2}\right)}{4 \pi \sqrt{2} \alpha \beta_{\mathrm{q}}^{2}},
$$

for a meson $\mathrm{V}$ of mass $M$. If $m_{\mathrm{h}}$ is close to the mass of $\mathrm{V}$, then eq. (27) may be an overestimation [27]. However, by studying the $b \bar{b}$ system, one still expects very strong constraints on $\beta_{\mathrm{b}}$ if $m_{\mathrm{h}}<9.4 \mathrm{GeV} / c^{2}$. If $m_{\mathrm{h}}=6 \mathrm{GeV}$ the ratio is $\sim 3 \times 10^{-2} / \beta_{\mathrm{b}}$.

The $\chi(2830)$ could conceivably be a Higgs [4] instead of an $\eta_{c}$. For this to be the case there must be large couplings to the Higgs so $\Gamma(\psi \rightarrow h \gamma)$ will be large. From eq. (27) we have

$$
\frac{\Gamma\left(\psi \rightarrow \mathrm{h}^{0} \gamma\right)}{\Gamma\left(\psi \rightarrow \mu^{+} \mu^{-}\right)}=\frac{1.36 \times 10^{-4}}{\beta^{2}} \lesssim 0.61 .
$$

Since $\operatorname{BR}\left(\psi \rightarrow \mu^{+} \mu^{-}\right) \approx 7 \%, \operatorname{BR}\left(\psi \rightarrow \mathrm{h}^{0} \gamma\right) \approx 4.3 \%$. The $\chi(2800)$ is seen by $\chi \rightarrow \gamma \gamma$; if the $\chi$ is a Higgs the rate is

$$
\frac{\Gamma(\mathrm{h} \rightarrow \gamma \gamma)}{\Gamma\left(\mathrm{h} \rightarrow \mu^{+} \mu^{-}\right)}=\frac{\alpha^{2} m_{\mathrm{h}}^{2}}{18 \pi^{2} M^{2}}\left(\sum_{\substack{\text { heavy } \\ \text { quarks }}} 9 e_{\mathrm{q}}^{4}+\sum_{\substack{\text { heavy } \\ \text { leptons }}}\right) \approx 1.1 \times 10^{-3},
$$

where "heavy" means $m_{\mathrm{q}, \ell} \gtrsim \frac{1}{2} M$. This small rate for $\gamma \gamma$ essentially eliminates the possibility that $\chi(2.83)$ is $\mathrm{h}^{0}$.

The main contributions to Higgs decay, if it were $\chi(2830)$, would be $\mathrm{H} \rightarrow \mathrm{s} \overline{\mathrm{s}}$ and $\mathrm{H} \rightarrow \mu^{+} \mu^{-}$. Since the quark mode has a factor of 3 from color and a factor $\left(m_{\mathrm{s}} / m_{\mu}\right)^{2}$ $=\frac{9}{4}$, it will dominate; we expect

$$
\Gamma_{\mathrm{h}}^{\text {tot }} \gtrsim\left(3\left(\frac{9}{4}\right)+1\right) \Gamma\left(\mathrm{h} \rightarrow \mu^{+} \mu^{-}\right)=8 \Gamma\left(\mathrm{h} \rightarrow \mu^{+} \mu^{-}\right),
$$

so $\mathrm{BR}(\mathrm{h} \rightarrow \gamma \gamma) \approx 0.14 \times 10^{-3}$. Then $\mathrm{BR}(\psi \rightarrow \gamma \mathrm{h}) \mathrm{BR}(\mathrm{h} \rightarrow \gamma \gamma) \approx 6 \times 10^{-6}$ in our model. This is more than an order of magnitude smaller than the reported rate (1.3 $\pm 10^{-4}$, which implies that even in our model with enhanced Higgs-fermion couplings it is not possible to interpret $\chi(2.83)$ as a Higgs boson.

The only way to get around these estimates would be to increase $\Gamma\left(\chi \rightarrow h^{0} \gamma\right)$ by having $\beta_{\mathrm{q}}<\beta_{\mu}$ by an order of magnitude, which seems very unlikely for the rele- 
vant quarks which are $s$ and heavier ones. For completeness, one should remark that to check experimentally on whether $\chi(2.8)$ were a Higgs one would want to find its decays in the ration of $\Gamma(\mathrm{h} \rightarrow$ strange particles $) / \Gamma\left(\mathrm{h} \rightarrow \mu^{+} \mu^{-}\right) / \Gamma(\mathrm{h} \rightarrow \gamma \gamma)$ to be about $7 / 1 / 10^{-3}$, with some phase-space suppression of the $7 / 1$.

For $\Upsilon$ instead of $\psi$, the rate for $\Upsilon \rightarrow h^{0} \gamma$ is changed by a factor $M^{2}(\Upsilon)-M^{2}(h)$, and the $\gamma \gamma$ branching ratio by a factor $M^{2}(\mathrm{~h})$. If $M(\mathrm{~h})-9 \mathrm{GeV}$, for example, the increase in the product of branching ratios is at least factor of 70 , if all $\beta$ 's are the same. In this situation the main decays of $h$ would be $\bar{c} \bar{c}$ and $\tau^{+} \tau^{-}$.

\subsection{Neutral Higgs effects in $e^{+} e^{-} \rightarrow \mu^{+} \mu^{-}$}

Experimenters hope to detect the presence of (s-channel) $\mathrm{Z}^{0}$ exchange in $\mathrm{e}^{+} \mathrm{e}^{-}$ $\rightarrow \mu^{+} \mu^{-}$by detecting a $\cos \theta$ term in the angular distribution of the outgoing muons [28]. This is not a parity violation; all one needs is an interference of two definite spin-parity exchanges to get a $\cos \theta$ terms. (The $\cos \theta$ term due to $Z$ exchange is due to the interference of $1^{+}$and $1^{-}$partial waves; such a term is also present in electromagnetic corrections to one photon exchange.) Therefore, one also expects a $\cos \theta$ term due to the presence of neutral Higgs exchange. Consider the calculation of $\mathrm{e}^{+} \mathrm{e}^{-} \rightarrow \mu^{+} \mu^{-}$at high energy which includes effects of $\mathrm{Z}^{0}$ and $\mathrm{h}^{0}$ exchange. Then, keeping only the dominant terms, the result is

$$
\begin{gathered}
\frac{\mathrm{d} \sigma}{\mathrm{d} \Omega_{\mathrm{cm}}}=\frac{\alpha^{2}}{4 s}\left[1+\cos ^{2} \theta+\frac{m_{\mathrm{e}}^{2} m_{\mu}^{2} s^{2}}{16 \beta_{\mathrm{e}}^{2} \beta_{\mu}^{2} \sin ^{4}\left(\theta_{\mathrm{W}}\right) m_{\mathrm{W}}^{4}\left[\left(m_{\mathrm{h}}^{2}-s\right)^{2}+m_{\mathrm{h}}^{2} \Gamma_{\mathrm{h}}^{2}\right]}\right] \\
+\frac{\alpha^{2} \cos \theta}{16 \sin ^{2} \theta_{\mathrm{W}} \cos ^{2} \theta_{\mathrm{W}}}\left[\frac{1}{s-m_{\mathrm{Z}}^{2}}-\frac{8 m_{\mathrm{e}}^{2} m_{\mu}^{2}\left(m_{\mathrm{h}}^{2}-s\right)}{\beta_{\mathrm{e}} \beta_{\mu} m_{\mathrm{Z}}^{2}\left[\left(m_{\mathrm{h}}^{2}-s\right)^{2}+m_{\mathrm{h}}^{2} \Gamma_{\mathrm{h}}^{2}\right]} \cdot\right]
\end{gathered}
$$

Let us first examine the $\cos \theta$ term in eq. (30). One would detect this term experimentally by measuring the asymmetry of the muon pairs. Note that for $s<m_{\mathrm{Z}}^{2}$ but $s>m_{\mathrm{h}}^{2}$ (we assume $m_{\mathrm{h}}<m_{\mathrm{Z}}$ as suggested hy eq. (8)), the $\mathrm{Z}$ and $\mathrm{h}^{0}$ contributions come in with opposite signs. Unfortunately, for any reasonable value of $\beta_{\mathrm{e}}$ and $\beta_{\mu}$, the $\mathrm{h}^{0}$ contribution is negligible for any $s$ (except for $s$ close to $m_{\mathrm{h}}^{2}$ ). The reason the effect is so small is because there is an extra factor of $m_{\mathrm{e}} m_{\mu}$ required to get $\gamma$-h ${ }^{0}$ interference (one factor of $m_{\mathrm{e}} m_{\mu}$ is due to the Higgs-fermion coupling). That is, for massless fermions, there is no $\gamma-h^{0}$ interference independent of the form of the Higgs-fermion coupling. The reason is that interference requires amplitudes with a net helicity of zero, and for the photon contribution these vanish when the fermion masses (see appendix $\mathrm{C}$ ) are zero.

Let us consider the case where $s \approx m_{\mathrm{h}}^{2}$ (i.e., sitting on top of the Higgs resonance). The contribution of the $\mathrm{h}^{0}$ term has a maximum at $s=m_{\mathrm{h}}^{2} \pm \Gamma_{\mathrm{h}} m_{\mathrm{h}}$. The term proportional to $\cos \theta$ is then approximately

$$
-\frac{\alpha^{2} \cos \theta}{16 m_{\mathrm{W}}^{2} \sin ^{2} \theta_{\mathrm{W}}}\left[1 \pm \frac{4 m_{\mathrm{e}}^{2} m_{\mu}^{2}}{\beta_{\mathrm{e}} \beta_{\mu} m_{\mathrm{h}}^{3} \Gamma_{\mathrm{h}}}\right],
$$


for $s \approx m_{\mathrm{h}}^{2} \ll<m_{Z}^{2}$. Now, if $m_{\mathrm{h}}$ is above $\bar{b} \overline{\mathrm{b}}$ threshold, then $\mathrm{h}^{0}$ will decay predominantly into $b \bar{b}$ pairs. In that case

$$
\Gamma_{\mathrm{h}}=\frac{3 G_{\mathrm{F}} m_{\mathrm{h}} m_{\mathrm{b}}^{2}}{4 \pi \sqrt{2} \beta^{2}}\left(1-\frac{4 m_{\mathrm{b}}^{2}}{m_{\mathrm{h}}^{2}}\right)^{3 / 2} .
$$

Otherwise, we need to use eq. (26) for $\mathrm{I}_{\mathrm{h}}$. Note that for $m_{\mathrm{h}}>2 m_{\mathrm{b}}$, we see that $\Gamma_{\mathrm{h}} / m_{\mathrm{h}} \approx 0.2$ for $\beta=0.015$. Thus, for small enough $\beta, \Gamma_{\mathrm{h}}$ may be quite large. However, to be optimistic, let us use the estimate we obtained from eq. (26): $\Gamma_{\mathrm{h}} / m_{\mathrm{h}} \approx$ $6 \times 10^{-6} / \beta^{2}$. Then, the term in brackets in eq. (31) becomes $1 \pm\left(2 \times 10^{-3} / m_{\mathrm{h}}^{4}\right)$ where $m_{\mathrm{h}}$ is in units of $\mathrm{GeV} / c^{2}$. We conclude that detection of the $\mathrm{h}^{0}$ in the $\cos \theta$ term is hopeless. Note that we have assumed $\beta_{\mathrm{e}}=\beta_{\mu}$ above; but our conclusion does not change for any reasonable values for the $\beta$ 's. The study of $\mathrm{e}^{+} \mathrm{e}^{-}$(apart from the experimental difficulties) would not be sufficient to observe the $h^{0}$ term.

Let us return to eq. (30) and examine the possibility of observing the Higgs meson in the total cross section. Now there are no extra factors of fermion masses; $\mathrm{h}^{0}$ and $\gamma$ contribute to different helicity amplitudes, which add incoherently. If we integrate over all angles, we find at resonance $\left(s=m_{\mathrm{h}}^{2}\right)$

$$
\sigma_{\text {tot }}\left(\mathrm{e}^{+} \mathrm{e}^{-}\right)=\frac{4 \pi \alpha^{2}}{3 s}\left[1+\frac{3 m_{\mathrm{e}}^{2} m_{\mu}^{2} m_{\mathrm{h}}^{2}}{64 \beta_{\mathrm{e}}^{2} \beta_{\mu}^{2} \sin ^{4}\left(\theta_{\mathrm{W}}\right) m_{\mathrm{W}}^{4} \Gamma_{\mathrm{h}}^{2}}\right] \text {. }
$$

If we assume $\beta_{\mathrm{e}}=\beta_{\mu} \equiv \beta$ and $\Gamma_{\mathrm{h}} / m_{\mathrm{h}} \approx 6 \times 10^{-6} / \beta^{2}$, then we obtain $1+\left(2 \times 10^{-6}\right)$ for the expression in brackets in eq. (33). The situation is not much better if we consider [2] $\sigma_{\text {tot }}\left(\mathrm{e}^{+} \mathrm{e}^{-} \rightarrow\right.$ anything). Using the same value for $\Gamma_{\mathrm{h}}$ and $\Gamma\left(\mathrm{h}^{0} \rightarrow \mu^{+} \mu^{-}\right)$ $=G_{\mathrm{F}} m_{\mathrm{h}} m_{\mu}^{2} / 4 \pi \sqrt{2} \beta^{2}$, we find that $\sigma\left(\mathrm{e}^{+} \mathrm{e}^{-} \rightarrow \mathrm{h} \rightarrow\right.$ anything $) / \sigma\left(\mathrm{e}^{+} \mathrm{e}^{-} \rightarrow \mu^{+} \mu^{-}\right) \approx 1.6$ $\times 10^{-3}$. Note that in both of these calculations, $\beta$ has dropped out of the final result; hence these results are just those of the standard one-Higgs model.

If we consider a model where $\beta_{\mathrm{e}} \neq \beta_{\mu}$, it is possible to get larger results. An example of such a situation appears in a paper by Kim and Segre [29]. Briefly, they construct a model where $\beta<<1$ for light fermions and $\beta=1$ for the heavier fermions. As a result, their Higgs bosons have rather small total widths, but substantial branching ratios into electrons and muons. Clearly, in such a model observable effects could occur in $\mathrm{e}^{+} \mathrm{e}^{-} \rightarrow \mu^{+} \mu^{-}$. Again, one should study the total cross section (eq. (33) rather than the asymmetry (eq. (31)). The latter is suppressed due to the extra factor of $m_{\mathrm{e}} m_{\mu}$ as discussed previously. Our conclusion is that effects due to neutral Higgs mesons are unlikely to be observable in $\mathrm{e}^{+} \mathrm{e}^{-}$physics at energies below the production of weak vector bosons.

\subsection{Higgs effects in Drell-Yan production of leptons}

The production of lepton pairs in hadron-hadron collisions is thought to occur via the reaction [30] $\mathrm{q} \overline{\mathrm{q}} \rightarrow \ell^{+} \ell^{-}$by one-photon exchange. We also expect a Higgs contribution; the discussion will be similar to the one given in subsect. 3.4. How- 
ever, there are a number of important differences. First, the charges of the quarks are fractional; hence the Higgs contribution relative to the one-photon exchange increases by a factor of $1 / e_{\mathrm{q}}^{2}$. Second, the quark mass will replace the electron mass which will increase the Higgs contribution here significantly as compared with $\mathrm{e}^{+} \mathrm{e}^{--} \rightarrow \mu^{+} \mu^{-}$. Third, there will be a color factor of $\frac{1}{3}$ multiplying the cross section.

One can now return to eq. (33) and derive results for the relative sizes of the $\mathrm{h}^{0}$ and $\gamma$ contributions to $\mathrm{q} \overline{\mathrm{q}} \rightarrow \mu^{+} \mu^{-}$. It has been remarked in the literature $[2,31]$ that the scattering of $u$ and $d$ quarks is not promising mechanism for producing Higgs. As remarked in ref. [31], in pp scattering, the dominant mechanism for Higgs production will be two-gluon scattering through a quark loop. Using our model, one would multiply the results of ref. [31] by $\beta^{-2}$, thus enhancing the production rate of Higgs. One still has to detect the Higgs once it has been produced. Although the $\mu^{+} \mu^{-}$mode is the easiest to detect experimentally, one can also look for $\mu^{ \pm} \mathrm{e}^{\mp}$ events which could signal $\tau^{+} \tau^{-}$production.

We would like to suggests one further possibility which could turn out to be an excellent place to look for a Higgs signal. Consider dimuon production in $\mathrm{K}^{+} \mathrm{N} \rightarrow$ $\mu^{+} \mu^{-}+\mathrm{X}$ or $\mathrm{K}^{0} \mathrm{~N} \rightarrow \mu^{+} \mu^{-}+\mathrm{X}$. It is crucial that the $\overline{\mathrm{s}}$ quark be the only antiquark which is also a valence quark. Then, one would expect that a significant part of the cross section would be due to $\overrightarrow{\mathrm{s}} \rightarrow \mu^{+} \mu^{-}$in the case of $\mathrm{K}^{+} \mathrm{N}$ and $\mathrm{d} \overline{\mathrm{d}} \rightarrow \mu^{+} \mu^{-}$in the case of $\mathrm{K}^{0} \mathrm{~N}$ ). Thus, in the case of an SU(3) symmetric sea, s $\overline{\mathrm{s}} \rightarrow \mu^{+} \mu^{-}$would account for half the dimuons. (Using a more realistic model for the sea, this ratio would be depressed by no more than a factor of two.) Consider now Higgs production in these reactions. Because the $\bar{s}$ antiquark is now valence and the mass of the strange quark is not negligible, we expect that Higgs production via $\bar{s}$ annihilation will be as large as the production by gluons. (In fact we believe that $\bar{s}$ annihilation actually dominates, but such a conclusion strongly depends on the quark distribution functions used.)

The above remarks are true for the standard model. If $\beta<1$, then we expect an enhancement in the production of Higgs. We present here some considerations which should give the reader a rough idea on the feasibility of detecting Higgs by such a method. From eq. (33), we obtain the peak height $\left(s=m_{\mathrm{h}}^{2}\right)$ :

$$
\rho \equiv \frac{\sigma\left(\overline{\mathrm{ss}} \rightarrow \mathrm{h}^{0} \rightarrow \mu^{+} \mu^{-}\right)}{\sigma\left(\overline{\mathrm{ss}} \rightarrow \gamma \rightarrow \mu^{+} \mu^{-}\right)}=\frac{3 m_{\mu}^{2} m_{\mathrm{s}}^{2}}{e_{\mathrm{q}}^{2} \alpha^{2}\left[\sum_{\mathrm{q}, \ell}\left(3 m_{\mathrm{q}}^{2}+m_{\ell}^{2}\right)\right]^{2}},
$$

where $e_{\mathrm{q}}=-\frac{1}{3}$ for an s-quark and $\alpha=\sqrt{2} G_{\mathrm{F}} m_{\mathrm{W}}^{2} \sin ^{2}\left(\theta_{\mathrm{W}}\right) / \pi \approx \frac{1}{137}$. We have assumed that all $\beta$ 's are equal and substituted for the Higgs width $\Gamma_{\mathrm{h}}$ using the generalization of eq. (26). (Near new thresholds, $\Gamma_{h}$ should be modified by appropriate phase-space factors; we ignore this here.) Note that as we remarked after eq. (33), $\beta$ cancels out of the ratio $\rho$. In a real experiment, we must take the experimental resolution $\Gamma_{\exp }$ into account and integrate over the peak using a Breit-Wigner shape. The signal one 
Table 2

Higgs production in ss annihilation

\begin{tabular}{|c|c|c|c|}
\hline$m_{\mathrm{h}}\left(\mathrm{GcV} / c^{2}\right)$ & Threshold & $\rho_{\exp }(\mathrm{MIN})$ & $\rho_{\exp }(\mathrm{MAX})$ \\
\hline 0.3 & $\mu^{+} \mu^{-}$ & $3.5 \times 10^{-2}$ & 0.3 \\
\hline $\begin{array}{l}0.5 \\
1.0 \\
3.0\end{array}$ & $\begin{array}{l}\bar{s} \\
\bar{s} \\
s \bar{s}\end{array}$ & $\begin{array}{l}7.5 \times 10^{-3} \\
1.5 \times 10^{-2} \\
4.5 \times 10^{-2}\end{array}$ & $\begin{array}{l}0.2 \\
1.5 \\
40\end{array}$ \\
\hline $\begin{array}{l}5.0 \\
9.0\end{array}$ & $\begin{array}{l}\bar{c} \bar{c}, \tau^{+} \tau^{-} \\
\bar{c} \bar{c} \tau^{+} \tau^{-}\end{array}$ & $\frac{6}{10^{-3^{*}} \times 10^{-4}}$ & $\begin{array}{l}0.8 \\
1.1\end{array}$ \\
\hline 20.0 & $\mathrm{~b} \overline{\mathrm{b}}$ & $3 \times 10^{-4}$ & 0.02 \\
\hline
\end{tabular}

Using eqs. (34) and (35) in the text, we calculate the Higgs signal-to-Drell Yan background noise for annihilation of sis (denoted by $\rho_{\exp }$ ). For illustrative purposes, we have assumed an experimental resolution of $\Gamma_{\exp }=100 \mathrm{MeV} . \rho_{\exp }(\mathrm{MIN})$ corresponds to $\beta=1$ and $\rho_{\exp }(\mathrm{MAX})$ corresponds to $\beta m_{\mathrm{h}}=0.1 \mathrm{GeV} / c^{2}$ (see the text following eq. (15)). If one chooses $m_{\mathrm{h}}$ close to a threshold, one must modify eq. (34) with an appropriate phase-space factor. In the "threshold" column below, we give the most massive fermions into which $\mathrm{h}$ can decay. We have taken $m_{\mathrm{s}}=150 \mathrm{MeV} / \mathrm{c}^{2}$.

"measures" in the laboratory then is

$$
\rho_{\exp } \equiv \frac{\Gamma_{\mathrm{h}} \rho}{\Gamma_{\exp }} \tan ^{-1} \frac{\Gamma_{\exp }}{\Gamma_{\mathrm{h}}}=\left\{\begin{array}{cl}
\frac{\pi \Gamma_{\mathrm{h}}}{2 \Gamma_{\exp }} \rho, & \Gamma_{\mathrm{h}} \ll \Gamma_{\exp }, \\
\rho & \Gamma_{\mathrm{h}} \gg \Gamma_{\exp }
\end{array} .\right.
$$

We interpret $\rho_{\exp }$ as a measure of the Higgs signal to Drell-Yan background noise


ground. The reader should decrease $\rho_{\exp }$ further to account for this background; this will depend of course on which beam is being used.

We present the results for $\rho_{\exp }$ in table 2 for a range of possible Higgs masses and $\beta$. We have chosen $\Gamma_{\exp }=100 \mathrm{MeV}$ for illustrative purposes. Note that although $\rho$ is independent of $\beta, \rho_{\exp }$ depends on $\beta$ through $\Gamma_{\mathrm{h}}$. We conclude that $\mathrm{K}^{+} \mathrm{N} \rightarrow$ $\mu^{+} \mu^{-}+\mathrm{X}$ is an excellent place to look for a Higgs particle of mass $\leqslant 10 \mathrm{GeV} / c^{2}$.

\subsection{Higgs effects in deep inelastic leptoproduction}

In our model with $\beta_{\mathrm{e}}=\beta_{\mu}$, one could find evidence for Higgs effects by studying breakdown of $\mu$-e univerality. As an example, we compare $\mu \mathrm{p} \rightarrow \mu \mathrm{X}$ with $\mathrm{ep} \rightarrow \mathrm{eX}$. To do this, consider the scattering of muon and electrons off quarks. The one-pho- 
ton exchange cross section is

$$
\frac{\mathrm{d} \sigma_{\gamma}}{\mathrm{d} Q^{2}}=\frac{4 \pi \alpha^{2} \mathrm{e}_{\mathrm{q}}^{2}}{Q^{4}}\left[\frac{1+(1-y)^{2}}{2}\right]
$$

where $\mathcal{c}_{\mathrm{q}}^{2}$ is the parton chargc squarcd, $Q^{2}$ is the four-momentum transfer squared and $y=1-E^{\prime} / E$ where $E,\left(E^{\prime}\right)$ is the initial (final) lepton energy in the lab system.

Let us compare this with the cross section due to Higgs $\left(\mathrm{h}^{0}\right)$ exchange. We may write the result in the following form:

$$
\frac{\mathrm{d} \sigma_{\mathrm{h}}}{\mathrm{d} Q^{2}}=\frac{G_{\mathrm{F}}^{2} m_{Q}^{2} m_{\mathrm{q}}^{2} x^{2} y^{2}}{8 \pi \beta_{Q}^{2} \beta_{\mathrm{q}}^{2}\left(m_{\mathrm{h}}^{2}+Q^{2}\right)^{2}},
$$

where $x=Q^{2} / y s$ and $\sqrt{ } s$ is the c.m. energy for the $\ell_{\mathrm{p}} \rightarrow \ell \mathrm{X}$ scattering. To get an idea of how large $\sigma_{\mathrm{h}}$ can be, let us divide eq. (37) by eq. (36), then

$$
\begin{gathered}
\frac{\mathrm{d} \sigma_{\mathrm{h}} / \mathrm{d} Q^{2}}{\mathrm{~d} \sigma_{\gamma} / \mathrm{d} Q^{2}} \leqslant \frac{G_{\mathrm{F}}^{2} m_{\ell}^{2} m_{\mathrm{q}}^{2}}{16 \pi^{2} \alpha^{2} e_{\mathrm{q}}^{2} \beta_{\ell}^{2} \beta_{\mathrm{q}}^{2}}, \\
=5.8 \times 10^{-8} m_{\ell}^{2} m_{\mathrm{q}}^{2} / \beta_{\ell}^{2} \beta_{\mathrm{q}}^{2},
\end{gathered}
$$

where we have taken an average squared charge $e_{\mathrm{q}}^{2}=\frac{5}{18}$ and masses are in $\mathrm{GeV}$ units. If we take $\beta_{\ell}=\beta_{\mathrm{q}}=0.015$, we see that the result is too small to be observed. On the other hand, if we put $\beta_{\mathrm{f}}=m_{\mathrm{f}} / 2 m_{\mathrm{W}}$ (with $m_{\mathrm{W}}=80 \mathrm{GeV} / c^{2}$ ) we obtain 38 for the above ratio. Then we must include the kinematical suppression factor

$$
\frac{x^{2} y^{2}}{1+(1-y)^{2}} \frac{Q^{2}}{\left(m_{\mathrm{h}}^{2}+Q^{2}\right)^{2}} \text {. }
$$

To estimate its effect, evaluate it at $x=y=\frac{1}{2}, Q^{2}=m_{\mathrm{h}}^{2}$; then it gives a reduction factor of $\frac{1}{80}$. This probably rules out the extreme case $\beta_{\mathrm{f}}=m_{\mathrm{f}} / 2 m_{\mathrm{W}}$ for both $\ell$ and $\mathrm{q}$, but gives little constraint if $\beta_{Q}=0.015$.

If $m_{\mathrm{f}} / \beta_{\mathrm{f}}$ is the same for all fermions, the Higgs contribution would not change the ratio of unity expected for $\sigma(\mathrm{eN} \rightarrow \mathrm{eX}) / \sigma(\mu \mathrm{N} \rightarrow \mu \mathrm{X})$. Otherwise since the Higgs contribution is proportional to $m_{\ell}^{2}$ it should give $\sigma(\mu \mathrm{N} \rightarrow \mu \mathrm{X})>\sigma(\mathrm{eN} \rightarrow \mathrm{eX})$.

\section{7. Energy level shifts}

In atomic systems the Higgs field will produce [32] a pure Yukawa potential

$$
V(r)=-c \frac{\mathrm{e}^{-m_{\mathbf{h}} r}}{r}
$$

which is the non-relativistic limit of $M=c\left(\bar{u}_{\mathrm{e}} u_{\mathrm{e}} \bar{u}_{\mathrm{p}} u_{\mathrm{p}}\right) /\left(k^{2}+m_{\mathrm{h}}^{2}\right)$ where

$$
c=\frac{g^{2}}{8 \pi m_{\mathrm{W}}^{2}} \beta_{\mathrm{e}} m_{\mathrm{e}} \sum_{\mathrm{q}} m_{\beta_{\mathrm{q}}}^{m_{\mathrm{q}}},
$$


and $q$ runs over all the particles in the nucleus. In particular the $2 \mathrm{P}_{3 / 2}$ to $2 \mathrm{~S}_{1 / 2}$ splitting this creates [33] is, for the $\mu$-He atom,

$$
\Delta E_{\mathrm{ps}}=c \frac{m_{\mathrm{h}}^{2}}{4 \alpha m_{\mu}}\left[1+\frac{m_{\mathrm{h}}}{2 \alpha m_{\mu}}\right]^{-4} \approx c \frac{4 \alpha^{3} m_{\mu}^{3}}{m_{\mathrm{h}}^{2}},
$$

assuming $m_{\mathrm{h}}$ \ン $2 \alpha m_{\mu}$. The difference between theory and experiment for this measurement is [34] less than the (theoretical) error, $8.7 \times 10^{-3} \mathrm{eV}$, so letting

$$
\sum_{\mathrm{q}} \frac{m_{\mathrm{q}}}{\beta_{\mathrm{q}}}=12 \frac{\bar{m}_{\mathrm{q}}}{\bar{\beta}_{\mathrm{q}}}
$$

$\bar{m}_{\mathrm{q}} \approx 5 \mathrm{MeV}$, we get

$$
2.4 \times 10^{-9} \mathrm{GeV}^{2}<\beta_{\mu} \bar{\beta}_{\mathrm{q}} m_{\mathrm{h}}^{2},
$$

which is much smaller than other limits. (At one time possible Higgs effects were considered to explain discrepancies in measurements in the X-ray spectrum of high- $Z$ muonic atoms. However, these were higher level transitions involving $f, p$ and $d$ states which are affected less by a high mass Higgs particle. The Higgs considered was less than $10 \mathrm{MeV}$ in mass.)

The $2 \mathrm{P}_{1 / 2}$ to $2 \mathrm{~S}_{1 / 2}$ transition in hydrogen has been measured [35] to within $8.3 \times 10^{-11} \mathrm{eV}$. Using the same formulae with $\mu \rightarrow$ e we have

$$
8.7 \times 10^{-9}(\mathrm{GeV})^{2}<\beta_{\mathrm{e}} \bar{\beta}_{\mathrm{q}} m_{\mathrm{h}}^{2} .
$$

For $\beta_{\mathrm{e}}=m_{\mathrm{e}} / m_{\mathrm{W}}, \bar{\beta}_{\mathrm{q}}=\bar{m}_{\mathrm{q}} / m_{\mathrm{W}}$, this gives an interesting limit for $m_{\mathrm{h}}, m_{\mathrm{h}}>4 \mathrm{GeV}$; it could provide a useful constraint on some theories. For $\beta_{\mathrm{e}}=\beta_{\mathrm{q}}=0.015$, it is not useful.

\section{Finding effects of the charged Higgs boson}

If the charged Higgs boson were light enough, it could be produced by $\mathrm{e}^{+} \mathrm{e}^{-} \rightarrow$ $\mathrm{H}^{+} \mathrm{H}^{-}$. This would lead to an increase in $R$ of $\frac{1}{4}$ of a unit above $\mathrm{H}^{+} \mathrm{H}^{-}$threshold. A good discussion of methods to observe this process in the context of the two-Higgs doublet model is given by Chang and Kim [36]. They suggest looking for peculiar final states (in particular four and six kaons in the final state) which could be associated with a small rise in $R$.

Unfortunately, if the mass estimates of eq. (8) are any guide, the $\mathrm{H}^{+}$will be too heavy to be produced by the next generation of $\mathrm{e}^{+} \mathrm{e}^{-}$machines. Thus, we will have to look for indirect ways to observe effects of the charged Higgs. We have already discussed in subsect. 2.2 the effect of charged Higgs exchange on the decay of pseudoscalar mesons. If $\beta_{\mathrm{e}}=\beta_{\mu}$, then the only hope for observing Higgs effects in a decay requires a situation where the non-relativistic quark model can be trusted (and the wave function at the origin is fairly well-known). In this section, we study other 




(a)

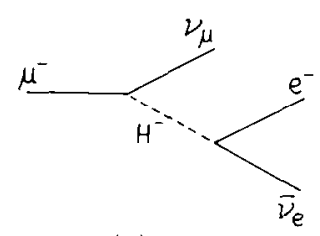

(b)

Iig. 4. Contributions to muon decay.

possibilities. In general, charged Higgs exchange can occur in any process in which $\mathrm{W}^{ \pm}$exchange occurs. Thus, the search for Higgs mesons becomes a search for scalar and pselidoscalar (charged) weak currents, kecping in mind the very special nature of the charged Higgs couplings.

\subsection{The decay $\mu \rightarrow e \nu \nu$}

The amplitude for the processes in fig. 4 is given by

$$
\begin{aligned}
M & =\sqrt{\frac{1}{2}} G_{\mathrm{F}} \bar{u}\left(k_{2}\right) \gamma_{\mu}\left(1+\gamma_{5}\right) u\left(p_{1}\right) \bar{u}\left(p_{2}\right) \gamma_{\mu}\left(1+\gamma_{5}\right) v\left(k_{1}\right) \\
& -\frac{m_{\mathrm{e}} m_{\mu}}{\beta_{\mathrm{e}} \beta_{\mu} m_{\mathrm{H}}^{2}} \bar{u}\left(k_{2}\right)\left(1-\gamma_{5}\right) u\left(p_{1}\right) \bar{u}\left(p_{2}\right)\left(1+\gamma_{5}\right) v\left(k_{1}\right) .
\end{aligned}
$$

For convenience, we apply a Fierz transformation. The result is

$$
\begin{aligned}
M & =\sqrt{\frac{1}{2}} G_{\mathrm{F}}\left[C_{V} \bar{u}\left(p_{2}\right) \gamma_{\mu} u\left(p_{1}\right) \bar{u}\left(k_{2}\right) \gamma_{\mu}\left(1+\gamma_{5}\right) v\left(k_{1}\right)\right. \\
& \left.+C_{\mathrm{A}} \bar{u}\left(p_{2}\right) i \gamma_{\mu} \gamma_{5} u\left(p_{1}\right) \bar{u}\left(k_{2}\right) i \gamma_{\mu}\left(1+\gamma_{5}\right) v\left(k_{1}\right)\right]
\end{aligned}
$$

where

$$
\begin{aligned}
& C_{\mathrm{V}}=1+m_{\mathrm{e}} m_{\mu} / \sqrt{2} \beta_{\mathrm{e}} \beta_{\mu} m_{\mathrm{H}}^{2}, \\
& C_{\mathrm{A}}=-1+m_{\mathrm{e}} m_{\mu} / \sqrt{2} \beta_{\mathrm{e}} \beta_{\mu} m_{\mathrm{H}}^{2} .
\end{aligned}
$$

Using the standard textbook results [37], we find

$$
\begin{aligned}
& \Gamma\left(\mu^{-} \rightarrow \mathrm{e}^{-} \bar{\nu}_{\mu} \nu_{\mathrm{e}}\right)=\frac{G_{\mathrm{F}}^{2} m_{\mu}^{5}}{192 \pi^{3}} \frac{1}{2}\left(C_{\mathrm{V}}^{2}+C_{\mathrm{A}}^{2}\right)\left(1+2 m_{\mathrm{e}} \eta / m_{\mu}\right), \\
& \eta=-\frac{1}{2}\left[\frac{C_{\mathrm{V}}^{2}-C_{\mathrm{A}}^{2}}{C_{\mathrm{V}}^{2}+C_{\mathrm{A}}^{2}}\right] \\
& \quad \approx-m_{\mathrm{e}} m_{\mu} / \sqrt{2} \beta_{\mathrm{e}} \beta_{\mu} m_{\mathrm{H}}^{2} .
\end{aligned}
$$

Another parameter $\xi$ which characterizes the electron asymmetry in polarized muon 
decay [37] is given by

$$
\begin{aligned}
\xi= & -2 C_{\mathrm{V}} C_{\mathrm{A}} /\left(C_{\mathrm{V}}^{2}+C_{\mathrm{A}}^{2}\right) \\
& \approx 1-m_{\mathrm{e}}^{2} m_{\mu}^{2} / \beta_{\mathrm{e}}^{2} \beta_{\mu}^{2} m_{\mathrm{H}}^{4} .
\end{aligned}
$$

The experimental numbers [37] are $\eta=-0.12 \pm 0.21$ and $\xi=0.973 \pm 0.014$. Note that for $m_{\mathrm{H}}>m_{\mathrm{W}}$, the Higgs contributions in eq. (42) and (43) are totally negligible if $\beta_{\mathrm{e}}=\beta_{\mu}=0.015$. However, $\beta_{\mathrm{f}}=m_{\mathrm{f}} / 2 m_{\mathrm{W}}$ is clearly ruled out (unless $m_{\mathrm{H}}>5 m_{\mathrm{W}}$ ). It is amusing to note that the experimental value for $\xi$ is more than one standard deviation away from the $\mathrm{V}-\mathrm{A}$ prediction of $\xi=1$. One should, however, include higher-order corrections (which can result in $\mathrm{V}+\mathrm{A}$ pieces) before one claims observation of a Higgs effect. Note that for our model, $\xi=1-2 \eta^{2}$ so $\eta$ is a more sensitive test.

Finally, note that the other Michel parameters $\rho=\delta=\frac{3}{4}$ renain unchanged bccause we have only left-handed neutrinos (except for terms on the order of $m_{\mu}^{2} / m_{\mathrm{W}}^{2}$ ). Better measurements of $\xi$ and $\eta$ are probably worthwhile.

\subsection{Beta decay}

Again, we will follow closely the analysis of the standard textbooks [37]. For beta decay, we approximate $H_{\text {eff }}$ as follows:

$$
\begin{aligned}
H_{\mathrm{eff}} & =\sqrt{\frac{1}{2}} G_{\mathrm{F}} \cos \theta_{\mathrm{C}}\left[\bar{p} \gamma_{\mu}\left(1+g_{\mathrm{A}} \gamma_{5}\right) n \bar{e} \gamma_{\mu}\left(1+\gamma_{5}\right) \nu\right. \\
& \left.+\bar{p}\left(g_{\mathrm{s}}+g_{\mathrm{p}} \gamma_{5}\right) n \bar{e}\left(1+\gamma_{5}\right) \nu\right],
\end{aligned}
$$

where $g_{\mathrm{A}} \approx 1.22$. If the interaction of the $\mathrm{H}^{+}$with the proton and neutron is assumed to be approximately the same as the one between the $\mathrm{H}^{+}$and the $\mathrm{u}$ and $\mathrm{d}$ quarks, then we can write

$$
\begin{aligned}
& g_{\mathrm{s}}=m_{\mathrm{e}}\left(m_{\mathrm{u}}-m_{\mathrm{d}}\right) / \beta_{\mathrm{ud}} \beta_{\mathrm{e} \nu} m_{\mathrm{H}}^{2}, \\
& g_{\mathrm{p}}=m_{\mathrm{e}}\left(m_{\mathrm{u}}+m_{\mathrm{d}}\right) / \beta_{\mathrm{ud}} \beta_{\mathrm{e} \nu} m_{\mathrm{H}}^{2} .
\end{aligned}
$$

Since $\beta$-decay is non-relativistic, we make a non-relativistic reduction on $H_{\text {eff }}$ (eq. (44)); and in this limit $\overline{\mathrm{p}} \gamma_{5} \mathrm{n} \rightarrow 0$. Hence, we will see no effect of $g_{\mathrm{p}}$. This makes the interpretation of the quark masses in eq. (45) much easier, since there is little difference between $m_{\mathrm{u}}-m_{\mathrm{d}}$ and $m_{\mathrm{p}}-m_{\mathrm{n}}$.

To see the effect of $g_{s}$, we look at the energy spectrum and angular distribution of the electron in $\beta$-decay. If we denote this distribution by $N\left(E_{\mathrm{e}}, \Omega_{\mathrm{e}}, \Omega_{\nu}\right)$ then [37],

$$
N\left(E_{\mathrm{e}}, \Omega_{\mathrm{e}}, \Omega_{\nu}\right) \propto \xi\left[E_{\mathrm{e}}+\lambda p_{\mathrm{e}} \cos \theta_{\mathrm{e} \nu}+b m_{\mathrm{e}}\right],
$$

where

$$
\xi=\left|M_{\mathrm{F}}\right|^{2}\left(1+g_{\mathrm{S}}^{2}\right)+\left|M_{\mathrm{GT}}\right|^{2} g_{\mathrm{A}}^{2},
$$




$$
\begin{aligned}
& \lambda \xi=\left|M_{\mathrm{F}}\right|^{2}\left(1-g_{\mathrm{s}}\right)^{2}-\frac{1}{3}\left|M_{\mathrm{GT}}\right|^{2} g_{\mathrm{A}}^{2}, \\
& b \xi=2\left|M_{\mathrm{F}}\right|^{2} g_{\mathrm{s}},
\end{aligned}
$$

where $M_{\mathrm{F}}$ and $M_{\mathrm{GT}}$ are the Fermi and Gamow-Teller matrix elements respectively. (For neutron $\beta$-decay, $\left|M_{\mathrm{F}}\right|=1$ and $\left|M_{\mathrm{GT}}\right|=\sqrt{ } 3$.) Note that $g_{\mathrm{s}}$ is even smaller than $\eta$ (defined in eq. (42)) because $m_{\mathrm{u}}-m_{\mathrm{d}} \approx-0.03 \mathrm{~m}_{\mu}$. Therefore, unless $\beta_{\mathrm{ud}}$ and very small (much smaller than $\beta=0.015$ ), the Higgs effects will be unobservable. As an example, consider the experimental measurement of $b$ in Fermi transitions. It is found [37] that $b=-0.02 \pm 0.09$. This allows one to obtain the limit $\beta_{\mathrm{e}} \beta_{\mathrm{ud}}$ $m_{\mathrm{H}}^{2}>3 \times 10^{-5} \mathrm{GeV}^{2}$. For $m_{\mathrm{H}}=160 \mathrm{GeV} / c^{2}$, we see that $\beta_{\mathrm{qq}}{ }^{\prime} \sim m_{\mathrm{q}} / m_{\mathrm{W}}$ is barely ruled out. This illustrates once again that one obtains very weak limits on the Higgs couplings from both $\beta$ and $\mu$ decays. It is useful to note, however, that one cannot have large charged Higgs-fermion couplings and a small $\mathrm{H}^{ \pm}$mass at the same time. In that case, both $\beta$ and $\mu$ decays can put some restrictions on the Higgs parameters. Furthermore, the $\mu$ decay results tend to be more stringent because $m_{\mu} \gg$ $m_{\mathrm{d}}-m_{\mathrm{u}}$.

For completeness, we note that for neutron $\beta$-decay

$$
\lambda=\frac{1-g_{\mathrm{A}}^{2}-g_{\mathrm{S}}^{2}}{g_{\mathrm{V}}^{2}+3 g_{\mathrm{A}}^{2}+g_{\mathrm{s}}^{2}} .
$$

Experimentally, one finds [37] that $\lambda=-0.091 \pm 0.039$. In fact, this is one of the ways (although not the best) one measures $g_{A}=0$ ). Therefore, a measurement of $\lambda$ is not useful to uncover a possible (but small) presence of $g_{\mathrm{s}}$.

One can also look at polarized neutron decay and various integrated transition rates for nucleon $\beta$-decay. Using current experimental numbers, one can obtain restrictions on $\beta_{\mathrm{e} v} \beta_{\mathrm{ud}} m_{\mathrm{H}}^{2}$ which are somewhat better than the one obtained from the measurement of $b$. However, our conclusions remain the same.

\subsection{Higgs effects in neutrino production}

There are no Higgs contributions to neutral current processes involving neutrinos (e.g., $\nu \mathrm{N} \rightarrow \nu \mathrm{X}$ ), because the neutrino is massless. However, there will be Higgs contributions to charged current processes. We examine one such example here.

Consider $\nu \mathrm{N} \rightarrow \mu^{-} \mathrm{X}$ in the framework of the parton model where $\mathrm{N}$ is an isoscalar target. That is, we calculate the elementary scatterings $\nu \mathrm{q} \rightarrow \mu^{-} \mathrm{q}^{\prime}$ and $\mu^{-} \mathrm{q}^{\prime}$ and then integrate over the distribution of quarks and antiquarks in the proton. Here we will take into account the possibility of $\mathrm{H}^{+}$exchange (as well as $\mathrm{W}^{+}$exchange). We can then derive the following formula for the cross section:

$$
\begin{aligned}
& \frac{\mathrm{d} \sigma^{\nu \mathrm{N}}}{\mathrm{d} x \mathrm{~d} y}=\frac{G_{\mathrm{F}}^{2} s}{2 \pi}\left\{\left[1+\frac{m_{\mu}^{2}\left(m_{\mathrm{u}}^{2}-m_{\mathrm{d}}^{2}(1-y)\right)}{s x \beta_{\mathrm{ud}} \beta_{\mu \nu} m_{\mathrm{H}}^{2}}+\frac{m_{\mu}^{2}\left(m_{\mathrm{u}}^{2}+m_{\mathrm{d}}^{2}\right) y^{2}}{4 \beta_{\mathrm{ud}}^{2} \beta_{\mu \nu}^{2} m_{\mathrm{H}}^{4}}\right] q(x)\right. \\
& \left.\quad+\left[(1-y)^{2}+\frac{m_{\mu}^{2}\left(m_{\mathrm{u}}^{2}(1-y)-m_{\mathrm{d}}^{2}\right)}{s x \beta_{\mathrm{ud}} \beta_{\mu \nu} m_{\mathrm{H}}^{2}}+\frac{m_{\mu}^{2}\left(m_{\mathrm{u}}^{2}+m_{\mathrm{d}}^{2}\right) y^{2}}{4 \beta_{\mathrm{ud}}^{2} \beta_{\mu \nu}^{2} m_{\mathrm{H}}^{4}}\right] \bar{q}(x)\right\},
\end{aligned}
$$


where $q(x)=x[u(x)+d(x)]$ (we ignore the other quark flavors). The effect of the Higgs contribution is to change the $x$ and $y$ dependence of the cross section. Let us first concentrate on the $y$ dependence. It will be convenient to compare eq. (49) with the following general expression for the cross section [38]:

$$
\frac{\mathrm{d} \sigma^{\nu \mathrm{N}}}{\mathrm{d} x \mathrm{~d} y} \sim \frac{\sigma_{\mathrm{L}}+\sigma_{\mathrm{R}}(1-y)^{2}+2 \sigma_{\mathrm{s}}(1-y)}{\sigma_{\mathrm{L}}+\sigma_{\mathrm{R}}+2 \sigma_{\mathrm{s}}}
$$

where $\sigma_{\mathrm{L}}, \sigma_{\mathrm{R}}$ and $\sigma_{\mathrm{s}}$ are the absorption cross sections for the scattering of left-handed, right-handed and scalar virtual $\mathrm{W}$ bosons respectively. In analogy with electroproduction, one defines $R \equiv 2 \sigma_{\mathrm{s}} /\left(\sigma_{\mathrm{L}}+\sigma_{\mathrm{R}}+2 \sigma_{\mathrm{s}}\right)$. In the naive parton model with only $\mathrm{W}$ exchange, $R=0$. Thus, the effect of the Higgs contribution is to give a nonzero value for $R$. Both low-energy (threshold) effects and scaling-violation corrections to the naive parton model also give small non-zero contributions to $R$ and at least the latter is non-negligible according to QCD predictions. If we note the identity $2(1-y) \equiv 1+(1-y)^{2}-y^{2}$, then we obtain

$$
R_{\text {Higgs }}=\frac{-m_{\mu}^{2}\left(m_{\mathrm{d}}^{2} q(x)-m_{\mathrm{u}}^{2} \bar{q}(x)\right)}{s x \beta_{\mathrm{ud}} \beta_{\mu \nu} m_{\mathrm{H}}^{2}}-\frac{m_{\mu}^{2}\left(m_{\mathrm{d}}^{2}+m_{\mathrm{u}}^{2}\right)(q(x)+\bar{q}(x))}{2 \beta_{\mathrm{ud}}^{2} \beta_{\mu \nu}^{2} m_{\mathrm{H}}^{4}},
$$

to be compared with the usual QCD predictions. In particular, $R_{\mathrm{Higgs}}$ is negative while $R_{\mathrm{QCD}}$ is positive and

$$
R_{\text {total }} \simeq R_{\text {Higgs }}+R_{\mathrm{QCD}} \text {. }
$$

so a significant cancellation can occur. If $m_{\mathrm{H}}$ is very large (as in eq. (8), then $R_{\mathrm{Higgs}}$ is negligible except possibly in the region where $x \rightarrow 0$. One should note that the kinematic assumptions which underlie the parton model break down as $x \rightarrow 0$. In addition, the term in eq. (49) or (51) which goes as $x^{-1}$ is multiplied by an extra product of fermion masses for similar reasons as those mentioned in subsect. 3.6 (this is a general feature of scalar-vector interference). In a deep inelastic process that current quark masses should be used. Thus, the situation here is similar to that of electroproduction; and in fact is even more hopeless if $m_{\mathrm{H}}$ is very large. We conclude that Higgs effects are unobservable in $\nu \mathrm{N} \rightarrow \mu^{-} \mathrm{X}$. By studying regions of very small $x$ at moderate energies one could perhaps put a lower bound on $m_{\mathrm{H}}$. We suspect however that given other sources for $R$ such a bound would be very weak.

\subsection{Parity violation in neutral currents due to Higgs}

Marciano and Sanda [39] have shown that radiative corrections which induce parity violation in the electron-nucleon interaction turn out to be much larger than 


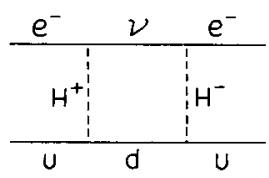

(a)

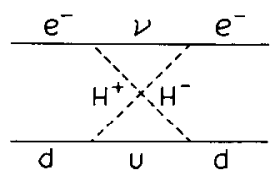

(b)

Fig. 5. Parity violation in the electron-nucleon neutral current induced by charged Higgs box diagrams. Note that these diagrams imply immediately that $C_{1 \mathrm{u}}^{\text {Higs }}=-C_{\mathbf{1 d}}^{\text {Higgs }}$. See text.

one might naively anticipate. The diagrams they studied which gave large effects were various box diagrams with two gauge bosons. With this in mind, we investigate the effect of box diagrams with two Higgs bosons. Since the neutral Higgs bosons are all parity conserving, we study the box diagram consisting of two charged Higgs (see fig. 5). These diagrams would contribute to parity violation in bismuth (Bi) and polarized electron-deuteron scattering $(\overrightarrow{e d} \rightarrow \mathrm{eX})$.

We will express our results in terms of the constants $C_{1 \mathrm{~d}}, C_{1 \mathrm{u}}, C_{2 \mathrm{~d}}$ and $C_{2 \mathrm{u}}$ defined by $[40]$

$$
\begin{aligned}
& H_{\mathrm{PV}}=\sqrt{\frac{1}{2}} G\left[C_{1 \mathrm{u}} \bar{e} \gamma_{\mu} \gamma_{5} e \bar{u} \gamma_{\mu} u+C_{1 \mathrm{~d}} e \gamma_{\mu} \gamma_{5} e \bar{d} \gamma_{\mu} d\right. \\
& \left.+C_{2 \mathrm{u}} \bar{e} \gamma_{\mu} e \bar{u} \gamma_{5} u+C_{2 \mathrm{~d}} \bar{e} \gamma_{\mu} e \bar{d} \gamma_{\mu} \gamma_{5} d\right] .
\end{aligned}
$$

In the Weinberg-Salam theory ( $Z^{0}$ exchange) one obtains

$$
\begin{aligned}
& C_{1 \mathrm{u}}=\frac{1}{2}\left(1-\frac{8}{3} \sin ^{2} \theta_{\mathrm{W}}\right), \\
& C_{1 \mathrm{~d}}=-\frac{1}{2}\left(1-\frac{4}{3} \sin ^{2} \theta_{\mathrm{W}}\right), \\
& C_{2 \mathrm{~d}}=\frac{1}{2}\left(1-4 \sin ^{2} \theta_{\mathrm{W}}\right), \\
& C_{2 \mathrm{~d}}=-\frac{1}{2}\left(1-4 \sin ^{2} \theta_{\mathrm{W}}\right) .
\end{aligned}
$$

Following the methods of ref. [39], we find the following results for the diagrams of fig. 5 (at small $Q^{2}$ ):

$$
\begin{aligned}
& C_{1 \mathrm{~d}}^{\mathrm{Higgs}}=\frac{G_{\mathrm{F}} m_{\mathrm{e}}^{2}\left(m_{\mathrm{u}}^{2}+m_{\mathrm{d}}^{2}\right)}{16 \sqrt{2} \pi^{2} m_{\mathrm{H}}^{2} \beta_{\mathrm{e} \nu}^{2} \beta_{\mathrm{ud}}^{2}}, \\
& C_{\mathrm{lu}}^{\mathrm{Higgs}}=-C_{\mathrm{ld}}^{\mathrm{Higgs}} .
\end{aligned}
$$

It is clear that given the limits of $\mu$-e universality in $\pi^{+}$decay (subsect. 2.2 ), the Higgs contribution to $C_{1 \mathrm{u}}$ and $C_{\mathbf{1 d}}$ must be negligible. However, we can also regard eq. (54) as providing limits on the Higgs parameters by studying $\overrightarrow{e d} \rightarrow \mathrm{eX}$. We note that the asymmetry in $\overrightarrow{e d} \rightarrow e X$ is predicted to be [41]

$$
A_{\mathrm{d}}(x, y) \sim\left(C_{1 \mathrm{u}}-\frac{1}{2} C_{1 \mathrm{~d}}\right)+\left(C_{2 \mathrm{u}}-\frac{1}{2} C_{2 \mathrm{~d}}\right)\left(\frac{1-(1-y)^{2}}{1+(1-y)^{2}}\right) \text {. }
$$


For the $y$ value measured by the SLAC parity violation experiment [40], the second term in eq. (55) is negligible. Hence, the asymmetry is proportional to $C_{1 \mathrm{u}}-\frac{1}{2} C_{1 \mathrm{~d}}$. If we regard the result of the SLAC experiment as confirming the Weinberg-Salam model, then any contribution to $C_{1 \mathrm{u}}$ and $C_{1 \mathrm{~d}}$ other than $\mathrm{Z}$ exchange must be small. Thus, $C_{1 \mathrm{u}}^{\mathrm{Higgs}}-\frac{1}{2} C_{1 \mathrm{~d}}^{\mathrm{Higgs}}=-1.5 C_{1 \mathrm{~d}}^{\mathrm{Higgs}}$ (where $C_{1 \mathrm{~d}}^{\mathrm{Higgs}}>0$; see eq. (54)) must be small compared to the WS prediction.

The above conclusion is useful when we consider parity violation in heavy atoms. Suppose we wish to "explain" the null result [42] in Bi using the Higgs box. It is possible to reduce the Weinberg-Salam prediction for parity violation in Bi by an appropriate choice of Higgs parameters. To see that, note that parity violation in $\mathrm{Bi}$ is proportional $10 Q_{\mathrm{W}} \equiv 2\left[C_{1 \mathrm{u}}(A+Z)+C_{1 \mathrm{~d}}(2 A-Z)\right] \approx 584\left(C_{1 \mathrm{u}}+1.15 C_{1 \mathrm{~d}}\right)$ for $Z=83, A=209$. Thus, using eqs. (53), (54) and $\sin ^{2} \theta_{\mathrm{W}}=\frac{1}{4}$, we see that

$$
C_{1 \mathrm{u}}+1.15 C_{1 \mathrm{~d}}=-0.22+0.15 C_{1 \mathrm{~d}}^{\mathrm{Higgs}} \text {. }
$$

Since $C_{1 \mathrm{~d}}^{\mathrm{Higgs}}>0$, one sees the possibility of reducing parity violation in Bi. Unfortunately, if we use the restriction on $C_{1 d}^{\mathrm{Higgs}}$ obtained from $\overrightarrow{\mathrm{ed}} \rightarrow \mathrm{eX}$, we conclude that the exchange of charged Higgs cannot be responsible for the null results in Bi.

We cannot resist mentioning one further amusing point. Suppose there were a contribution for which $C_{1 \mathrm{u}} \sim m_{\mathrm{u}}, C_{1 \mathrm{~d}} \sim m_{\mathrm{d}}$. Then for the $\overrightarrow{\mathrm{eN}} \rightarrow$ eX experiment, which measures $C_{1 \mathrm{u}}-\frac{1}{2} C_{1 \mathrm{~d}}$ we get a contribution proportional to $m_{\mathrm{u}}-\frac{1}{2} m_{\mathrm{d}}$, which is essentially zero for current quark masses; such a contribution would automatically not affect the SLAC experiment. Conversely, for the Bi experiment, we would ohtain $C_{1 \mathrm{u}}+1.15 C_{1 \mathrm{~d}}=-0.22+k\left(m_{\mathrm{u}}+1.15 m_{\mathrm{d}}\right)$ which could reduce the Bi prediction.

A neutral Higgs box in a $C P$-violating theory would couple proportional to $m_{\mathrm{u}}^{2}$ or $m_{\mathrm{d}}^{2}$, essentially satisfying these conditions.

If the situation with parity violation in $\mathrm{eN}$ interactions clears up soon and is in accord with the standard theory, as most people expect, then the above remarks in this section are at best of pedagogical interest. If the situation should remain puzzling, they may help provide clues to what is happening.

Finally, let us note that Higgs box diagrams could contribute in other processes (e.g., $\nu_{\mu}$ e scattering and $\mathrm{e}^{+} \mathrm{e}^{-} \rightarrow \mu^{+} \mu^{-}$). The arguments in this section suggest that such effects will be small.

\section{5. $K_{L}^{0}-K_{S}^{0}$ mass difference and flavor-changing neutral currents}

For completeness, we note that one should always make sure that a given model of weak interactions does not upset the standard calculations [43] of the $\mathrm{K}_{\mathrm{L}}^{0}-\mathrm{K}_{\mathrm{S}}^{0}$ mass difference and rare $\mathrm{K}$ meson decay widths. In our model, one would consider box diagrams where one or more $W$ boson is replaced by a charged Higgs boson. Grifols [44] has considered the effect of box diagrams with one Higgs boson and one $\mathrm{W}$ on the $\mathrm{K}_{\mathrm{L}}^{0}-\mathrm{K}_{\mathrm{S}}^{0}$ mass difference in an $\mathrm{SU}(2)_{\mathrm{L}} \times \mathrm{SU}(2)_{\mathrm{R}} \times \mathrm{U}(1)$ gauge model. 
He finds that for $m_{\mathrm{H}} \approx m_{\mathrm{W}}$, the coupling of $\mathrm{H}$ to fermions could be as large as $30 \%$ of $g$ without upsetting any low-energy weak interactions. In our notation, this would mean that $0.3 \beta_{\mathrm{sd}}>m_{\mathrm{s}} / 2 m_{\mathrm{W}}$ or $\beta_{\mathrm{sd}} \gtrsim 10^{-2}$ (if we take $m_{\mathrm{s}} \approx 500 \mathrm{MeV} / c^{2}$ ). This result is consistent with our results of sect. 2 .

In general we will have flavor-changing neutral currents induced by box diagrams, and they could be comparable with the usual contributions to $\mathrm{K}_{\mathrm{L}}^{0} \rightarrow \mathrm{K}_{\mathrm{S}}^{0}$ or $\mathrm{K}^{0} \rightarrow$ $\mu^{+} \mu^{--}$but not large enough to disrupt conventional views.

In a sense the contribution of scalar currents in box diagrams giving a $\mathrm{V}, \mathrm{A}$ theory is related to the earlier attempts [45] to do this in general. We conclude that the restrictions on Higgs couplings do not allow the Higgs to play the role of such scalars.

\section{Discussion}

In this paper we have studied some implications for experiments of a scheme in which Higgs-fermion couplings can be large. While fermion and vector boson masses still both arise from spontaneous symmetry breaking, we assume their widely different mass scales are set by different scales of vacuum expectation values (vev's) of Higgs bosons. We constructed one explicit model in which two different vev's are present, one $(v)$ giving mass to fermions and one $(V)$ to vector bosons. Then there is a small parameter $\beta=v / V$, and Higgs-fermion couplings are of order $g m_{\mathrm{f}} / 2 \beta m_{\mathrm{W}}$. That is the Higgs-fermion couplings are enhanced by a factor $\beta^{-1}$ over that of the standard model. If one found that $\beta_{\mathfrak{f}}=m_{\mathfrak{f}} / 2 m_{\mathrm{W}}$ for a given Higgs-fermion coupling that would correspond to a coupling equal to $g$. The only experiment which clearly gives a relevant constraint is $(g-2)$ for the muon. Even this constraint is model dependent and could be relaxed in more complicated situations. One finds under certain assumptions regarding Higgs masses, that $\beta_{\mu} \geq 10 m_{\mu} / m_{\mathrm{W}}$. Furthermore, one has suggestions from $\pi^{+}$leptonic decay and comparisons of $\mathrm{eN} \rightarrow \mathrm{eX}$ with $\mu \mathrm{N} \rightarrow \mu \mathrm{X}$ that $\beta_{\mathrm{e}} \ll \beta_{\mu} \ll 1$ is unlikely.

We have constructed the simplest model where there is only one parameter $\beta$ for all fermions. Should it turn out that $\beta \approx 1$, then one should consider wrong our premise that the mass scales of fermions and vector bosons are set by different scales of vev's. It appears that $\beta>10^{-2}$; such a value might still be consistent with our premise. Already we have evidence for fermions such that $m_{\mathbf{f}} / m_{\mathrm{W}} \approx 0.05$. Our sense of what values $\beta$ will take is surely not sound enough to give such an interpretation with any confidence. Further, some experiments will soon be good enough to see the effects of strongly coupled Higgs if they are present, or could already be analyzed with such a question in mind. Consequently, we have given a number of experimental tests in the text.

Indeed, it is somewhat surprising that existing experiments do not limit the Higgs coupling more. They allow the muon-Higgs coupling to be about 75 times the Weinberg-Salam value, and restrict other couplings even less, if $h^{0}$ is the only "light" 
Higgs (i.e., the only Higgs boson with mass significantly less than $m_{\mathrm{W}}$ ).

If it turns out that $\beta$ is significantly less than 1 , then we have a number of interesling phenomena which could arise from the Higgs sector. These have been discussed in the text and summarized in table 1 . Here, we would like to mention two other interesting possibilities. The first concerns the problem of the axion [46]. (The axion is a pseudoscalar much lighter than the pseudoscalar $\mathrm{H}^{0}$ of our model.) The two-Higgs model we have presented here does not contain any particle which could be an axion. This is simply due to our choice of the Higgs-fermion interaction (i.e., choice of $g_{1}=0$ in eq. (B.22)). One could add more doublets and arrange for an axion by appropriately choosing the Higgs-fermion couplings. In the standard model of the axion, the coupling of axions to fermions is of order $m_{f} / 2 m_{W}$. The result of this fact is that the axion is expected to have a very light mass (probably less than an electron mass). It appears that such an axion is excluded by experiment [47]. By constructing a model where the coupling of axions to fermions is of order $m_{\mathrm{f}} / 2 \beta m_{\mathrm{W}}$, however, one finds $[46,48]$ that the mass of the axion is proportional to $\beta+\beta^{-1}$. The result is that axions are now heavier (with a mass in the GeV's). If this turns out to be the case, one may now turn to the tests given in sect. 3 for the detection of the axion. Furthermore, the axion will then contribute to $(g-2)_{\mu}$ and does so with a sign opposite to that of the $h^{0}$ contribution, perhaps significantly weakening the constraint on $\beta_{\mu}$.

A second interesting effect (assuming $\beta<<1$ ) could be weak interaction effects at very high energies. Should one of the heavy Higgs particles in our model have a mass of order $1 \mathrm{TeV} / c^{2}$, then we have a possibility of a breakdown of the weak interaction perturbation $[5,6]$ theory. A signal for this effect might be the appearance of unusual large weak interacting effects at very high energies (at the next generation of $p p$ and $\bar{p} p$ colliding beam machines when $\sqrt{ }_{s} \sim 1 \mathrm{TeV}$ ). Such effects are screened off in the standard model $[5,7]$ by a factor $m_{f} / m_{W}$, while in our model these phenomena would be larger by a factor $\beta^{-1}$. For possible ways of detecting them, see ref. [49].

If nature turns out to be similar to the standard model, it will be very difficult to detect the Higgs boson, either directly or by its effects. It is important, however, to note that whereas the standard model is increasingly well confirmed in almost every aspect, the Higgs sector remains fairly unconstrained. By studying experimental consequences of alternative Higgs sectors we may be able to point to an experiment which will find a Higgs-related effect, or eventually it may become clear that the standard theory with the simplest Higgs sector is the better alternative.

Two of us (H.E.H. and G.L.K) are grateful to S.D. Ellis and E. Henley for their hospitality at the Summer Institute in Weak Interaction Theory, where part of this work was done. In addition, one of us (H.F.H.) would like to thank J.D. Jackson for discussions and express appreciation to R.N. Cahn and Y. Kazama for conversations related to appendix A, and another (G.L.K.) appreciates stimulating remarks from J. Leveille. 


\section{Appendix A}

How to obtain the interactions of the physical Higgs particles

Since we use in some detail the properties of several of the physical Higgs states in our model, and they are not conventional, we provide a pedagogical review of the general method here and then apply it to the two-Higgs model in appendix B.

Step 1: Start with a complex multiplet of Higgs fields. Then we will need representation matrices for the generators of the gauge group which satisfy

$$
\left[T_{a}, T_{b}\right]=i f_{a b c} T_{c} .
$$

We define

$$
L_{a}=-i T_{\mathrm{a}} .
$$

The matrices $L_{a}$ can be chosen to be real by doubling the dimension of the representation. Hence, from now on we will assume that the $I_{a}$ are real $N \times N$ matrices.

Step 2: Construct the vector meson mass matrix. The gauge bosons gain mass through the Higgs mechanism. The kinetic energy term in the Higgs lagrangian is

$$
-\varrho=\left(D_{\mu} \phi\right)^{+} D_{\mu} \phi,
$$

where $D_{\mu}=\partial_{\mu}+g_{a} L_{a} W_{\mu}^{a}$ ( $W_{\mu}^{a}$ are the vector boson fields). To obtain the mass matrix, replace $\phi$ by its vacuum expectation value $v$ (note that both $\phi$ and $v$ are vectors on which the $L_{a}$ operate). The mass matrix is then read off by comparing with $\frac{1}{2} M_{a b}^{2} W^{a} W^{b} ;$ the result is

$$
M_{a b}^{2}=2\left(g_{a} L_{a} v\right)^{\mathrm{T}}\left(g_{b} L_{b} v\right)
$$

(where the superscript $\mathrm{T}$ means matrix transpose). Note that there is no sum over $a$ or $b$ in eq. (A.4). We have given a subscript to the coupling $g$ to account for the possibility that the gauge group may be a product of simple groups (or $U(1)$ pieces), each with a different coupling.

Step 3: Diagonalize the mass matrix $M^{2}$. Denote the orthogonal diagonalizing matrix by $U$.

Step 4: Form a linear combination of the $L_{a}$ as follows:

$$
\tilde{L}_{b}=\sum_{a} g_{a} U_{a b} L_{a}
$$

Step 5: We may now write down the Goldstone bosons (which will give mass to vector bosons in the theory). For convenience, let us assemble all the Higgs multi- 
plets in the theory into one column vector:

$$
\phi=\left[\begin{array}{c}
\phi_{1}^{1} \\
\phi_{1}^{2} \\
\cdot \\
\cdot \\
\phi_{2}^{1} \\
\cdot \\
\cdot
\end{array}\right],
$$

where we have denoted $\phi_{i}^{j}$ as the $j$ th real component of the Higgs multiplet $\phi_{i}$. By assumption (see remark after eq. (A.2)), all $\phi_{i}^{i}$ are real. We also construct matrices $L$ in block form

$$
\tilde{L}_{a}=\left[\begin{array}{ccc}
\tilde{L}_{a}^{1} & 0 \\
0 & \widetilde{L}_{a}^{2} & \\
& & \cdot \\
& & \cdot
\end{array}\right]
$$

where $\widetilde{L}_{a}^{i}$ are the linear combination of representation matrices (eq. (A.5)) corresponding to $\phi_{i}$. Then the Goldstone bosons (denoted by $\psi_{a}$ ) are just those linear combination of fields $\phi_{i}^{j}$ given by

$$
\psi_{a}=\left(\check{L}_{a} V\right)^{\mathrm{T}}[\phi-v]
$$

where $\phi$ is now given by eq. (A.6) and $v \equiv\langle\phi\rangle$. Note that the number of independent Goldstone bosons is equal to the number of broken generators, i.e., the number of $\widetilde{L}_{a}$ such that $\widetilde{L}_{a} v \neq 0$.

Step 6: The physical Higgs particles (also linear combinations of the $\phi_{i}^{i}$ ) are orthogonal to the $\psi_{a}$. That is, if we write $\psi_{a}=\Sigma C_{i j} \phi_{i}^{j}$ and $\chi_{a}=\Sigma d_{i j} \phi_{i}^{j}$ (where the $\chi_{a}=$ $\Sigma d_{i j} \phi_{i}^{i}$ (where the $\chi_{a}$ are the physical Higgs particles), then

$$
\sum_{i j} C_{i j} d_{i j}=0
$$

This does not uniquely determine the proper linear combination of physical Higgs bosons (by proper, we mean particles of definite mass). To obtain this information, 
one must examine the Higgs mass matrix

$$
m_{i j}^{2}=\left(\frac{\partial^{2} V}{\partial \phi_{i} \partial \phi_{j}}\right)_{\phi=v}
$$

(where we use the notation $\phi_{i}$ to mean the $i$ th row of the column vector $\phi$ ). Since the Goldstone bosons are already known, one may simplify the task of extracting masses and the proper linear combinations which correspond to the physical Higgs.

Step 7: Once the proper linear combinations which correspond to the physical Higgs are known, it is straightforward to take an interaction term from the original lagrangian involving $\phi$ and work out the interactions of the physical Higgs partticles.

\section{Appendix B}

A sample model: two Higgs doublets

We will apply the methods of appendix A to the following model. Consider a model with two complex $y=1$ scalar doublets:

$$
\phi_{1} \equiv\left[\begin{array}{l}
\phi_{1}^{+} \\
\phi_{1}^{0}
\end{array}\right], \quad \phi_{2} \equiv\left[\begin{array}{l}
\phi_{2}^{+} \\
\phi_{2}^{0}
\end{array}\right],
$$

in an $\mathrm{SU}(2) \times U(1)$ gauge theory. For simplicity, we will impose exact $C P$ conservation and require the Higgs lagrangian to be invariant under the discrete symmetry $\phi_{1} \rightarrow-\phi_{1}, \phi_{2} \rightarrow+\phi_{2}$. Then the most general Higgs potential has the form [50]

$$
\begin{aligned}
& V\left(\phi_{1}, \phi_{2}\right)=\lambda_{1}\left(\phi_{1}^{+} \phi_{1}-V^{2}\right)^{2}+\lambda_{2}\left(\phi_{2}^{+} \phi_{2}-v^{2}\right)^{2} \\
& \quad+\lambda_{3}\left[\left(\phi_{1}^{+} \phi_{1}-V^{2}\right)+\left(\phi_{2}^{+} \phi_{2}-V^{2}\right)\right]^{2} \\
& \quad+\lambda_{4}\left[\left(\phi_{1}^{+} \phi_{1}\right)\left(\phi_{2}^{+} \phi_{1}\right)-\left(\phi_{1}^{+} \phi_{2}\right)\left(\phi_{2}^{+} \phi_{1}\right)\right]+\lambda_{6}\left(\operatorname{Im}\left(\phi_{1}^{+} \phi_{2}\right)\right)^{2},
\end{aligned}
$$

where the $\lambda_{i}$ are positive. (A term $\lambda_{5}\left[\operatorname{Re}\left(\phi_{1}^{+} \phi_{2}\right)-a_{1} a_{2}\right]^{2}$ is forbidden by our discrete symmetry.) We have written the potential in the above form so that the vacuum expectation values can be obtained by inspection:

$$
\phi_{1}=\left(\begin{array}{l}
0 \\
V
\end{array}\right), \quad \phi_{2}=\left(\begin{array}{l}
0 \\
v
\end{array}\right) .
$$

(Note that $V$ and $v$ are relatively real if $C P$ invariance holds.) The vector boson masses are easily computed using eq. (A.4):

$$
\begin{aligned}
& m_{\mathrm{W}}^{2}=\frac{1}{2} g^{2}\left(v^{2}+V^{2}\right), \\
& m_{\mathrm{Z}}=m_{\mathrm{W}} / \cos \theta_{\mathrm{W}} .
\end{aligned}
$$


Note that eq. (B.4b) is true in any $\mathrm{SU}(2) \times \mathrm{U}(1)$ theory with any number of Higgs doublets but no other Higgs multiplets [13]. Now, $\phi_{1}$ and $\phi_{2}$ consist of eight degrees of freedom. There will be three Goldstone bosons to give mass to the $W^{ \pm}$ and $Z^{0}$; thus five physical Higgs particles will remain. To demonstrate the methods of appendix A, we will write out the matrices $L_{a}$ (eq. (A.2)) explicitly. The generators of $\mathrm{SU}(2) \times \mathrm{U}(1)$ in the doublet representation are $\frac{1}{2} \sigma, \frac{1}{2} y(y$ is the $2 \times 2$ identity matrix) which satisfy $Q=\frac{1}{2}\left(\sigma_{3}+y\right)$. Multiply by $-i$ to get the matrices $L_{a}$, then go to the real representation $(4 \times 4$ matrices). The result is

$$
\begin{aligned}
& L_{1}=\frac{1}{2}\left[\begin{array}{rrrr}
0 & 0 & 0 & 1 \\
0 & 0 & -1 & 0 \\
0 & 1 & 0 & 0 \\
-1 & 0 & 0 & 0
\end{array}\right], \quad L_{2}=\frac{1}{2}\left[\begin{array}{rrrr}
0 & 0 & 1 & 0 \\
0 & 0 & 0 & 1 \\
-1 & 0 & 0 & 0 \\
0 & -1 & 0 & 0
\end{array}\right], \\
& L_{3}=\frac{1}{2}\left[\begin{array}{rrrr}
0 & 1 & 0 & 0 \\
-1 & 0 & 0 & 0 \\
0 & 0 & 0 & -1 \\
0 & 0 & 1 & 0
\end{array}\right], \quad L_{4}=\frac{1}{2}\left[\begin{array}{rrrr}
0 & 1 & 0 & 0 \\
-1 & 0 & 0 & 0 \\
0 & 0 & 0 & 1 \\
0 & 0 & -1 & 0
\end{array}\right] .
\end{aligned}
$$

Let us next find the matrix $U$ which diagonalizes the vector meson mass matrix $M^{2}$ (eq. (A.4)). The charged sector $(a=1,2)$ is already diagonal so we concentrate on the neutral sector $(a=3,4)$. The neutral mass matrix is

$$
U^{-1}\left[\begin{array}{ll}
m_{\mathrm{Z}}^{2} & 0 \\
0 & 0
\end{array}\right] U=\frac{v^{2}+V^{2}}{2}\left[\begin{array}{cc}
g^{2} & -g g^{\prime} \\
-g g^{\prime} & g^{\prime 2}
\end{array}\right],
$$

where $g$ and $g^{\prime}$ are the $\mathrm{SU}(2)$ and $\mathrm{U}(1)$ couplings respectively. $g^{2} / 8 m_{\mathrm{W}}^{2}=\sqrt{\frac{1}{2}} G_{\mathrm{F}}$. If we denote $\tan \theta_{\mathrm{W}}=g^{\prime} / g$, then we regain eq. (B.4) with

$$
U=\left[\begin{array}{rr}
\cos \theta_{\mathrm{W}} & \sin \theta_{\mathrm{W}} \\
-\sin \theta_{\mathrm{W}} & \cos \theta_{\mathrm{W}}
\end{array}\right) \text {. }
$$

Hence, the $\widetilde{L}_{a}$ defined by eq. (A.5) become (for $a=3,4$ ):

$$
\begin{aligned}
\widetilde{L}_{3} & =g L_{3} \cos \theta_{\mathrm{W}}-g^{\prime} L_{4} \sin \theta_{\mathrm{W}}, \\
\widetilde{L}_{4} & =g L_{3} \sin \theta_{\mathrm{W}}+g^{\prime} L_{4} \cos \theta_{\mathrm{W}} \\
& =g g^{\prime}\left(L_{3}+L_{4}\right) /\left(g^{2}+g^{\prime 2}\right)^{1 / 2} .
\end{aligned}
$$

But $\tilde{L}_{4}$ is just proportional to the charge operator $Q$; it is the unbroken generator. Note that since the charged sector is already diagonal, $\widetilde{L}_{1}=L_{1}$ and $\widetilde{L}_{2}=L_{2}$. Thus, we can read off the Goldstone bosons from eq. (A.8) (which we are free to normalize):

$$
G^{ \pm}=\phi_{1}^{ \pm} \cos \beta+\phi_{2}^{ \pm} \sin \beta
$$




$$
2^{1 / 2} G^{0}=\cos \beta \operatorname{Im} \phi_{1}^{0}+\sin \beta \operatorname{Im} \phi_{2}^{0},
$$

where

$$
\tan \beta=v / V,
$$

and we have used the notation of eq. (B.1). There are only two charged physical Higgs; hence they must be orthogonal to $G^{ \pm}$:

$$
H^{ \pm}=-\phi_{1}^{ \pm} \sin \beta+\phi_{2}^{ \pm} \cos \beta \text {. }
$$

Similarly, the three physical neutral Higgs particles must all be orthogonal to $G^{0}$, that is, linear combinations of $-\sin \beta \operatorname{Im} \phi_{1}^{0}+\cos \beta \operatorname{Im} \phi_{2}^{0}, \operatorname{Re}\left(\phi_{1}^{0}-V\right)$ and $\operatorname{Re}\left(\phi_{2}^{0}-v\right)$. (Note that all physical Higgs particles have zero vacuum expectation values). The proper linear combinations are obtained by studying the Higgs mass matrix (see eq. (A.10)). Let us write eq. (B.1) as

$$
\phi_{1} \rightarrow\left[\begin{array}{c}
\phi_{1} \\
\phi_{2} \\
\phi_{3} \\
\phi_{4}
\end{array}\right], \quad \phi_{2} \rightarrow\left[\begin{array}{c}
\phi_{5} \\
\phi_{6} \\
\phi_{7} \\
\phi_{8}
\end{array}\right]
$$

Consider first the charged sector (indices $1,2,5,6$ ). The mass matrix for $\mathrm{H}^{+}, \mathrm{G}^{+}$is

$$
2 \lambda_{4}\left[\begin{array}{cc}
v^{2} & -v V \\
-v V & V^{2}
\end{array}\right] .
$$

The non-zero eigenvalue is the mass of $\mathrm{H}^{+}$:

$$
m_{\mathrm{H}^{+}}^{2}=2 \lambda_{4}\left(v^{2}+V^{2}\right) \text {. }
$$

One can check that the eigenvectors of eq. (B.13) reproduce the correct form for $G^{ \pm}$and $H^{ \pm}$.

The mass matrix for the neutral sector (indices $3,4,7,8$ ) is

$$
\left[\begin{array}{llll}
8 V^{2}\left(\lambda_{1}+\lambda_{3}\right) & 0 & 8 \lambda_{3} v V & 0 \\
0 & 2 \lambda_{6} v^{2} & 0 & -2 \lambda_{6} v V \\
8 \lambda_{3} v V & 0 & 8 v^{2}\left(\lambda_{2}+\lambda_{3}\right) & 0 \\
0 & -2 \lambda_{6} v V & 0 & 2 \lambda_{6} V^{2}
\end{array}\right]
$$

The three physical Higgs obtained are

$$
\begin{aligned}
& 2^{-1 / 2} H^{0}=-\sin \beta \operatorname{Im} \phi_{1}^{0}+\cos \beta \operatorname{Im} \phi_{2}^{0}, \\
& 2^{-1 / 2} \phi^{0}=\cos \alpha\left(\operatorname{Re} \phi_{1}^{0}-V\right)+\sin \alpha\left(\operatorname{Re} \phi_{2}^{0}-v\right), \\
& 2^{-1 / 2} h^{0}=-\sin \alpha\left(\operatorname{Re} \phi_{1}^{0}-V\right)+\cos \alpha\left(\operatorname{Re} \phi_{2}^{0}-v\right) .
\end{aligned}
$$


The mass of $\mathrm{H}^{0}$ is given by

$$
m_{\mathrm{H}^{0}}^{2}=2 \lambda_{6}\left(v^{2}+V^{2}\right) \text {. }
$$

The angle $\alpha$ and the masses of $\phi^{0}$ and $h^{0}$ are complicated functions of $v, V$ and the $\lambda_{i}$

In this paper, we are interested in the situation where $v \ll V$ so that $\tan \beta$ is small (see eq. (B.10)). Hence, $\tan \beta \approx \beta$ and in this approximation we find

$$
\begin{aligned}
& \alpha \approx v \lambda_{3} / V\left(\lambda_{1}+\lambda_{3}\right) \approx \lambda_{3} \beta /\left(\lambda_{1}+\lambda_{3}\right), \\
& m_{\phi}^{2} \approx 8 V^{2}\left(\lambda_{1}+\lambda_{3}\right)\left(1+\alpha^{2}\right), \\
& m_{\mathrm{h}}^{2} \approx 8 V^{2}\left(\lambda_{1}+\lambda_{3}\right)\left(\lambda_{1} \lambda_{2}+\lambda_{1} \lambda_{3}+\lambda_{2} \lambda_{3}\right) \alpha^{2} / \lambda_{3}^{2} .
\end{aligned}
$$

Note that eq. (B.17) implies that $\alpha \leqslant \beta$ (since the $\lambda_{i}$ are positive). Therefore $m_{\mathrm{h}}$ is the only Higgs mass proportional to $\beta$ which is very small (by assumption). Actually, the Higgs masses are in reality free parameters because there is no constraint on the $\lambda_{i}$. Suppose, however, that all the $\lambda_{i}$ were of order $g^{2}$. Then, the Higgs masses would be determined. For example, if we choose $\lambda_{i}=g^{2}$ for all $i$, we would find (in the limit that $\beta$ is small)

$$
\begin{aligned}
& m_{\mathrm{H}}^{2} \approx 2 g^{2} V^{2} \approx 4 m_{\mathrm{W}}^{2} \approx\left(160 \mathrm{GeV} / c^{2}\right)^{2} \\
& m_{\phi}^{2} \approx 16 g^{2} V^{2} \approx 32 m_{\mathrm{W}}^{2} \approx\left(450 \mathrm{GeV} / c^{2}\right)^{2} \\
& m_{\mathrm{h}}^{2} \approx 12 g^{2} V^{2} \beta^{2} \approx 24 m_{\mathrm{W}}^{2} \beta^{2} \approx\left(400 \beta \mathrm{GeV} / c^{2}\right)^{2}
\end{aligned}
$$

where we have used $m_{\mathrm{W}} \approx 80 \mathrm{GeV} / c^{2}$. Note that by choosing $\lambda_{4}=\lambda_{6}, m_{\mathrm{H}^{+}}=m_{\mathrm{H}^{0}}$; we denote the common mass by $m_{\mathrm{H}}$. Sometimes we will use these to guide our thinking.

Finally, we are ready to obtain the coupling of the physical Higgs particles to fermions. Consider the most general coupling of Higgs to the leptons. We assume all the neutrinos are massless. Then, denoting

$$
\begin{aligned}
& \ell=\frac{1}{2}\left(1+\gamma_{5}\right)\left[\begin{array}{l}
\nu_{\mathrm{e}} \\
e^{-}
\end{array}\right], \\
& e_{\mathrm{R}}=\frac{1}{2}\left(1-\gamma_{5}\right) e^{-},
\end{aligned}
$$

the most general Higgs-lepton coupling is

$$
-\mathcal{L}=g_{1}\left(\bar{e}_{\mathrm{R}} \phi_{1}^{+} \ell+\bar{\ell} \phi_{1} e_{\mathrm{R}}\right)+g_{2}\left(\bar{e}_{\mathrm{R}} \phi_{2}^{+} \ell+\bar{\ell} \phi_{2} e_{\mathrm{R}}\right) \text {. }
$$

The mass of the electron is obtained by replacing the Higgs fields with their vacuum expectation values:

$$
m_{\mathrm{e}}=g_{1} V+g_{2} v .
$$

Our philosophy here is to attribute the existence of two very different mass scales of the theory (the vector boson masses and the fermion masses) to the exis- 


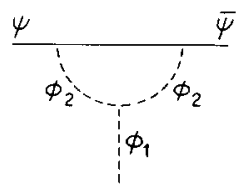

(a)

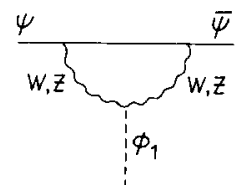

(b)

lig. 6. Perturbation terms inducing $\bar{\psi} \phi_{1} \psi$ couplings.

tence of two very different vacuum expectation values $v$ and $V$. If we choose $g_{1} \equiv 0$ in eq. (B.22), then we have succeeded in our goal: fermion masses will be proportional to $v$ whereas vector boson masses (see eq. (B.4a)) will be proportional to $\mathrm{V}>\boldsymbol{v}$. This can be done by maintaining the symmetry $\phi_{1} \rightarrow-\phi_{1}$ beyond the Higgs potential.

The symmetry $\phi_{1} \rightarrow-\phi_{1}$ is broken by the vev of the $\phi_{1}$; this means that unless some other symmetry prevents it there will be an induced coupling of $\phi_{1}-\left\langle\phi_{1}\right\rangle$ to fermions. The diagrams which induce a $g_{1} \bar{\psi} \psi \phi_{1}$ interaction are shown in fig. 6 . The $\phi_{1} \phi_{2} \phi_{2}$ coupling is approximately $g m_{\mathrm{H}}^{2} / m_{\mathrm{W}}$, the $\bar{\psi} \psi \phi_{2}$ coupling is $g m_{\mathrm{f}} / \beta m_{\mathrm{W}}$, and there is a factor $m_{\mathbf{f}} / m_{\mathbf{H}}^{2}$ from the propagator in the loop integral, so the induced strength from fig. $6 \mathrm{a}$ is $g^{3} m_{\mathrm{f}}^{3} / \beta M_{\mathrm{W}}^{3}$ which is very small. For the $\mathrm{W}$ or $\mathrm{Z}$ the coupling to the fermions is $g$ and the coupling to the $\phi_{1}$ is $g m_{\mathrm{H}}^{2} / m_{\mathrm{W}}$. A factor $m_{\mathrm{f}} / m_{\mathrm{W}}^{2}$ comes from the integral so we finally have for fig. $6 \mathrm{~b} \mathrm{~g}^{3} m_{\mathrm{f}} / m_{\mathrm{W}}\left(M_{\mathrm{H}}^{2} / m_{\mathrm{W}}^{2}\right)$. This is small compared to $g m_{\mathrm{f}} / m_{\mathrm{W}}$ (the smallest coupling of a Higgs to a fermion) unless $g^{2} m_{\mathrm{H}}^{2}>$; $m_{\mathrm{W}}^{2}$. These induced couplings depend on the existence of the second Higgs because without it the fermion mass is zero and the diagrams vanish. Thus, we impose the following condition on our theory: fermions do not couple to $\phi_{1}$. Note that such a theory will not have flavor changing neutral currents in the Higgs sector [15]. Using eqs. (B.22), (B.23) we may write out $\phi_{2}$ in terms of the physical Higgs and Goldstone bosons (eqs. (B.9) - (B.11), (B.16), (B.17)). We then obtain the following interaction between physical Higgs particles and leptons:

$$
\begin{aligned}
& \frac{m_{\mathrm{e}} g}{2 \beta m_{\mathrm{W}}}\left[\bar{e} e \phi^{0} \sin \alpha+\bar{e} e h^{0} \cos \alpha-i \bar{e} \gamma_{5} e H^{0} \cos \beta\right] \\
& -\frac{m_{\mathrm{e}} g}{2 \sqrt{2} \beta m_{\mathrm{W}}}\left[\bar{\ell}\left(1+\gamma_{5}\right) \nu_{\mathrm{Q}} H^{-}+\bar{\nu}_{\mathrm{Q}}\left(1-\gamma_{5}\right) \ell H^{+}\right]
\end{aligned}
$$

where we have assumed that $\alpha \leqslant \beta<<1$. Note that the couplings of the neutral Higgs are parity conserving as they must be in a $C P$-invariant theory (neutral flavor conserving scalars which violate $P$ must also violate $T$ ). From eq. (B.24), we see that $\phi^{0}$ and $\mathrm{h}^{0}$ are $\mathrm{O}^{+}$particles, whereas $\mathrm{H}^{0}$ is a $0^{-}$particle. Furthermore, the coupling of $\mathrm{H}^{ \pm}$is parity violating as it must be since the neutrinos are left handed. Finally, other lepton doublets will couple analogously to the electron doublet. 
Since we have assumed all neutrinos are massless, there will be no mixing in the lepton sector.

We now turn to the quarks. We again assume that the quarks couple only to $\phi_{2}$. This will give mass to the charge $-\frac{1}{3}$ quarks. In addition, we introduce $\widetilde{\phi}_{2}=i \sigma_{2} \phi_{2}^{*}$ which is a $y=-1$ doublet. This gives mass to the charge $\frac{2}{3} q u a r k s$. Proceeding in a manner analogous to the one for leptons, we obtain the most general coupling of the four quarks to $\phi_{2}$ :

$$
\begin{aligned}
& -\mathscr{L}=\frac{m_{\mathrm{d}} \cos \theta_{\mathrm{C}}}{v}\left(\bar{d}_{\mathrm{R}} \phi_{2}^{+} q_{1 \mathrm{~L}}+\bar{q}_{1 \mathrm{~L}} \phi_{2} d_{\mathrm{R}}\right)+\frac{m_{\mathrm{S}} \cos \theta_{\mathrm{C}}}{v}\left(\bar{s}_{\mathrm{R}} \phi_{2}^{+} q_{2 \mathrm{~L}}+q_{2 \mathrm{~L}} \phi_{2} s_{\mathrm{R}}\right) \\
& +\frac{m_{\mathrm{S}} \sin \theta_{\mathrm{C}}}{v}\left(\bar{s}_{\mathrm{R}} \phi_{2}^{+} q_{1 \mathrm{~L}}+\bar{q}_{1 \mathrm{~L}} \phi_{2} s_{\mathrm{R}}\right)-\frac{m_{\mathrm{d}} \sin \theta_{\mathrm{C}}}{v}\left(\bar{d}_{\mathrm{R}} \phi_{2}^{+} q_{2 \mathrm{~L}}+\bar{q}_{2 \mathrm{~L}} \phi_{2} d_{\mathrm{R}}\right) \\
& \quad+\frac{m_{\mathrm{u}}}{v}\left(\bar{u}_{\mathrm{R}} \tilde{\phi}_{2}^{+} q_{1 \mathrm{~L}}+q_{1 \mathrm{~L}} \tilde{\phi}_{2} u_{\mathrm{R}}\right)+\frac{m_{\mathrm{c}}}{v}\left(\bar{c}_{\mathrm{R}} \tilde{\phi}_{2}^{+} q_{2 \mathrm{~L}}+\bar{q}_{2 \mathrm{~L}} \tilde{\phi}_{2} c_{\mathrm{R}}\right)_{[}
\end{aligned}
$$

for doublets

$$
q_{1}=\left[\begin{array}{c}
u \\
d \cos \theta_{\mathrm{C}}+s \sin \theta_{\mathrm{C}}
\end{array}\right], \quad q_{2}=\left[\begin{array}{c}
c \\
s \cos \theta_{\mathrm{C}}-d \sin \theta_{\mathrm{C}}
\end{array}\right] .
$$

Again, we substitute the physical Higgs and Goldstone bosons. The end result is the following Higgs-quark coupling which we write out explicity for the first quark doublet $\left(q_{1}\right)$ only

$$
\begin{aligned}
& \frac{m_{\mathrm{u}} g}{2 \beta m_{\mathrm{W}}}\left[\bar{u} u \phi^{0} \sin \alpha+\bar{u} u h^{0} \cos \alpha+\bar{i} \bar{u} \gamma_{5} u H^{0} \cos \beta\right] \\
& \quad+{ }^{m_{\mathrm{d}} g}\left[\bar{d} d \phi^{0} \sin \alpha+\bar{d} d h^{0} \cos \alpha \quad i \bar{d} \gamma_{5} d H^{0} \cos \beta\right] \\
& \quad+\frac{g \cos \theta_{\mathrm{C}}}{2 \sqrt{2} \beta m_{\mathrm{W}}}\left\{\bar{u}\left[m_{\mathrm{u}}\left(1+\gamma_{5}\right)-m_{\mathrm{d}}\left(1-\gamma_{5}\right)\right] d H^{+}+\text {h.c. }\right\} \\
& \quad+\frac{g \sin \theta_{\mathrm{C}}}{2 \sqrt{2} \beta m_{\mathrm{W}}}\left\{\bar{u}\left[m_{\mathrm{u}}\left(1+\gamma_{5}\right)-m_{\mathrm{s}}\left(1-\gamma_{5}\right)\right] s H^{+}+\text {h.c. }\right\}
\end{aligned}
$$

where we have assumed that $\beta<<1$. Note that in the limit of $m_{\mathbf{u}}=0$ and $\sin \theta_{\mathbf{C}}=0$ we obtain exactly the result of eq. (B.24) as we should.

\section{Appendix C}

$f \dot{f} \rightarrow f^{\prime} \bar{f}^{\prime}$ arnplitudes

It is useful to write out the helicity amplitudes for $f \dot{\mathrm{f}} \rightarrow \mathrm{f}^{\prime} \overline{\mathrm{f}}^{\prime}$ reactions. Then for any combination of $\gamma, Z, h$ contributions, one can easily find cross sections and 


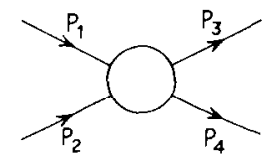

lig. 7. Momentum conventions.

interference terms, and examine the origin of interferences.

We give results in the c.m.s., with momenta defined as in fig. 7 , in the limit of large $s$.

Define

$$
\begin{aligned}
& M^{1}=\bar{u}_{3} \gamma_{\mu}\left(V^{\prime}+A^{\prime} \gamma_{5}\right) v_{4}\left(\delta_{\mu \nu}+Q_{\mu} Q_{\nu} / m_{\mathrm{V}}^{2}\right) \bar{v}_{2} \gamma_{\nu}\left(V+A \gamma_{5}\right) u_{1}, \\
& M^{0}=\bar{u}_{3}\left(S^{\prime}+P^{\prime} \gamma_{5}\right) v_{4} \bar{v}_{2}\left(S+P \gamma_{5}\right) u_{1} .
\end{aligned}
$$

Note the kinematical relations $s=-\left(p_{1}+p_{2}\right)^{2}, t=-\left(p_{3}-p_{1}\right)^{2}, u=-\left(p_{3}-p_{2}\right)^{2}$, $t \approx-\frac{1}{2} s(1-\cos \theta), y \equiv-t / s, s+t=s(1-y), y=\frac{1}{2}(1-\cos \theta), 1-y=\frac{1}{2}(1+\cos \theta)$, $k^{2}=\frac{1}{4} s-m_{\mathrm{f}}^{\prime 2}, p^{2}=\frac{1}{4} s-m_{\mathrm{f}}^{2}$. Cross sections summed and averaged over spins are given by

$$
\mathrm{d} \sigma / \mathrm{d} t=\sum|M|^{2} / 64 \pi s^{2}, \quad \mathrm{~d} \Omega=4 \pi \mathrm{d} t / s .
$$

The 16 helicity amplitudes are given in table 3 .

\begin{tabular}{|c|c|c|}
\hline 1234 & $M^{1}$ & $M^{0}$ \\
\hline $\begin{array}{l}+++ \\
++--\end{array}$ & $\begin{array}{r}4 m m^{\prime}\left[-V V^{\prime} \cos \theta+A A^{\prime}\left(1-s / 4 m_{Y}^{2}\right)\right] \\
-4 m m^{\prime}\left[+V V^{\prime} \cos \theta+A A^{\prime}\left(1-s / 4 m_{V}^{2}\right)\right]\end{array}$ & $\begin{array}{l}s(S-P)\left(S^{\prime}+P^{\prime}\right) \\
s(S-P)\left(S^{\prime}-P^{\prime}\right)\end{array}$ \\
\hline--++ & $-4 m m^{\prime}\left[+V V^{\prime} \cos \theta+A A^{\prime}\left(1-s / 4 m_{V}^{2}\right)\right]$ & $s(S+P)\left(S^{\prime}+P^{\prime}\right)$ \\
\hline--- & $4 m m^{\prime}\left[-V V^{\prime} \cos \theta+A A^{\prime}\left(1-s / 4 m_{V}^{2}\right)\right]$ & $s(S+P)\left(S^{\prime}-P^{\prime}\right)$ \\
\hline+-+- & $-s\left(V^{\prime}-A^{\prime}\right)(V-A)(1+\cos \theta)$ & 0 \\
\hline+--+ & $-s\left(V^{\prime}+A^{\prime}\right)(V-A)(1-\cos \theta)$ & 0 \\
\hline-++- & $-s\left(V^{\prime}-A^{\prime}\right)(V+A)(1-\cos \theta)$ & 0 \\
\hline-+-+ & $-s\left(V^{\prime}+A^{\prime}\right)(V+A)(1+\cos \theta)$ & 0 \\
\hline+++ & $2 \sqrt{s}\left(V^{\prime}-A^{\prime}\right) V m \sin \theta$ & 0 \\
\hline$++\ldots+$ & $-2 \sqrt{s}\left(V^{\prime}+A^{\prime}\right) V m \sin \theta$ & 0 \\
\hline+-++ & $2 \sqrt{s} V^{\prime}(V-A) m^{\prime} \sin \theta$ & 0 \\
\hline$+\ldots$ & $2 \sqrt{s} V^{\prime}(V-A) m^{\prime} \sin \theta$ & 0 \\
\hline-+++ & $-2 \sqrt{s} V^{\prime}(V+A) m^{\prime} \sin \theta$ & 0 \\
\hline-+-- & $-2 \sqrt{s} V^{\prime}(V+A) m^{\prime} \sin \theta$ & 0 \\
\hline--+- & $2 \sqrt{s}\left(V^{\prime}-A^{\prime}\right) V m \sin \theta$ & 0 \\
\hline$--\ldots+$ & $-2 \sqrt{s}\left(V^{\prime}+A^{\prime}\right) V m \sin \theta$ & 0 \\
\hline
\end{tabular}

Table 3

Helicity amplitudes 


\section{References}

[1] S. Weinberg, Phys. Rev. Lett. 19 (1967) 1264;

A. Salam, in Elementary particle physics, ed. N. Svartholm (Almqvist and Wiksell, Stockholm, 1968) p. 367.

[2] J. Ellis, M.K. Gaillard and D.V. Nanopoulos, Nucl. Phys. B106 (1976) 292.

[3] M.K. Gaillard, The Higgs particle, CERN preprint TH. 2461 (1978).

[4] F. Wilczek, Phys. Rev. Lett. 39 (1977) 1304.

[5] M. Veltman, Acta Phys. Polon. B8 (1977) 475.

[6] B.W. Lee, C. Quigg and H.B. Thacker, Phys. Rev. Lett. 38 (1977) 883; Phys. Rev. D16 (1977) 1519.

[7] H.E. Haber and G.L. Kane, Nucl. Phys. B144 (1978) 525.

[8] L.M. Sehgal, Aachen preprint PITHA-102 (1978), Proceedings of Neutrino'78 Conf., to be published.

[9] J.J. Sakurai, UCLA preprint 78/TEP/18 (1978) (invited paper presented at the Topical Conf. on Neutrino physics at accelerators, Oxford, July 3-7, 1978).

[10] C.Y. Prescott et al., SLAC-PUB-2148 (July, 1978).

[11] R.R. Lewis and W.L. Williams, Phys. Lett. 59B (1975) 70.

[12] P.Q. Hung and J.J. Sakurai, Phys. Lett. 69B (1977) 323.

[13] D.A. Ross and M. Veltman, Nucl. Phys. B95 (1975) 135.

[14] S. Weinberg, Phys. Rev. Lett. 37 (1976) 657.

[15] S.L. Glashow and S. Weinberg, Phys. Rev. D15 (1977) 1958.

[16] S. Weinberg, The problem of mass, A Festschrift in honor of I.I. Rabi (New York Academy of Sciences, 1977).

[17] H.D. Politzer, Nucl. Phys. B117 (1976) 397;

H. Georgi and H.D. Politzer, Phys. Rev. D14 (1976) 1829.

[18] A.H. Mueller, Phys. Rev. D18 (1978) 3705;

R.K. Ellis, et al., Nucl. Phys. B152 (1979) 285.

[19] M.A. Shifman, A.I. Vainshtein, and V.I. Zakharov, Phys. Lett. 78B (1978) 443.

[20] J.P. Leveille, Nucl. Phys. B137 (1978) 63.

[21] P. Ramond and G.G. Ross, Constraints on Higgs couplings from axionatics, Cal Tech preprint CALT 68-674 (1978).

[22] S. Narison, The anomalous magnetic moment of charged heavy lepton, Centre de Physique Theorique CNRS 77/p. 957.

[23] R. Van Royen and V.F. Weisskopf, Nuovo Cim. 50A (1967) 617; 51A (1967) 583.

[24] R. Barbieri, R. Kogerler, Z. Kunszt and R. Gatto, Nucl. Phys. B105 (1976) 125 ; J.D. Jackson, Lectures on the new particles, Proc. SLAC Summer Inst. on Particle physics, 1976, SLAC-198 (1976).

[25] S.D. Drell, Nuovo Cim. 11 (1959) 603;

D.A. Geffen and B.-L. Young, Phys. Rev. Lett. 15 (1965) 316;

C. Quigg and J.D. Jackson, UCRL-18487 (1968), unpublished.

[16] J. Fischer et al., Phys. Lett. 73B (1978) 364; Particle Data Group, Phys. Lett. 75B (1978) 1.

[17] P.H. Frampton, W.W. Wada, Phys. Rev. D19 (1979) 271.

[28] T. Himel et a1., Phys. Rev. Lett. 41 (1978) 449.

[29] J.E. Kim and G. Segre, Phys. Lett. 78B (1978) 75.

[30] S.D. Drell and T.-M. Yang, Phys. Rev. Lett. 25 (1970) 316; Ann. of Phys. 66 (1971) 578.

[31] H. Georgi, S.L. Glashow, M. Machacek and D.V. Nananopoulos, Phys. Rev. Lett. 40 (1978) 692.

[32] S.L. Adler, R.F. Dashen and S.B. Treiman, Phys. Rev, D10 (1974) 3782.

[33] R. Barbieri, Phys. Lett. 56B (1975) 266. 
[34] G. Carboni et al., Nuovo Cim. 34A (1976) 266.

[35] S.R. Lundeen and F.M. Pipkin, Phys. Rev. Lett. 34 (1976) 493.

[36] L.N. Chang and J.E. Kim, University of Pennsylvania preprint UPR-0094T (1978).

[37] R.E. Marshak, Riazuddin and C.P. Ryan, Theory of weak interactions in particle physics, (Wiley-Interscience, New York, 1969);

E.D. Commins, Weak interactions (McGraw-Hill, New York, 1973).

[38] C.Il. Llcwellyn Smith, Phys. Reports 3 (1972) 261.

[39] W.J. Marciano and A.I. Sanda, Phys. Rev. D17 (1978) 3055.

[40] R.N. Cahn and G.L. Kane, Phys. Lett. 71 B (1977) 348.

[41] R.N. Gahn and F.I. Gilman, Phys. Rev. D17 (1978) 1313.

[42] L.C. Lewis et al., Phys. Rev. Lett. 39 (1977) 795; P. Baird et al., Phys. Rev. Lett. 39 (1977) 798.

[43] M.K. Gaillard and B.W. Lee, Phys. Rev. D1 0 (1974) 897.

[44] J.A. Grifols, SLAC-PUB-2108 (April, 1978).

[45] G. Segre, Phys. Rev. Lett. 33 (1974) 1244.

[46] S. Weinberg, Phys. Rev. Lett. 40 (1978) 223;

F. Wilczek, Phys. Rev. Lett, 40 (1978) 279.

[47] T.W. Donnelly et al., Phys. Rev. D18 (1978) 1607.

[48] W.A. Bardeen and S.-H.H. Tye, Phys. Lett. 74B (1978) 229.

[49] G.L. Kane, Invited talk at the Int. Conf. on Cosmic rays and particle physics above 10 TeV, Bartol Research Institute, Oct., 1978.

[50] H. Georgi, A model of soft $C P$ violation, Harvard preprint HUTP-78/A010 (1978).

[51] J. Calmet, S. Narison, M. Perrottet and F. de Rafael, Rev. Mod. Phys. 49 (1977) 21. 